\title{
1 Allopregnanolone mediates affective switching through 2 modulation of oscillatory states in the basolateral amygdala
}

3

5

6

7

8

9

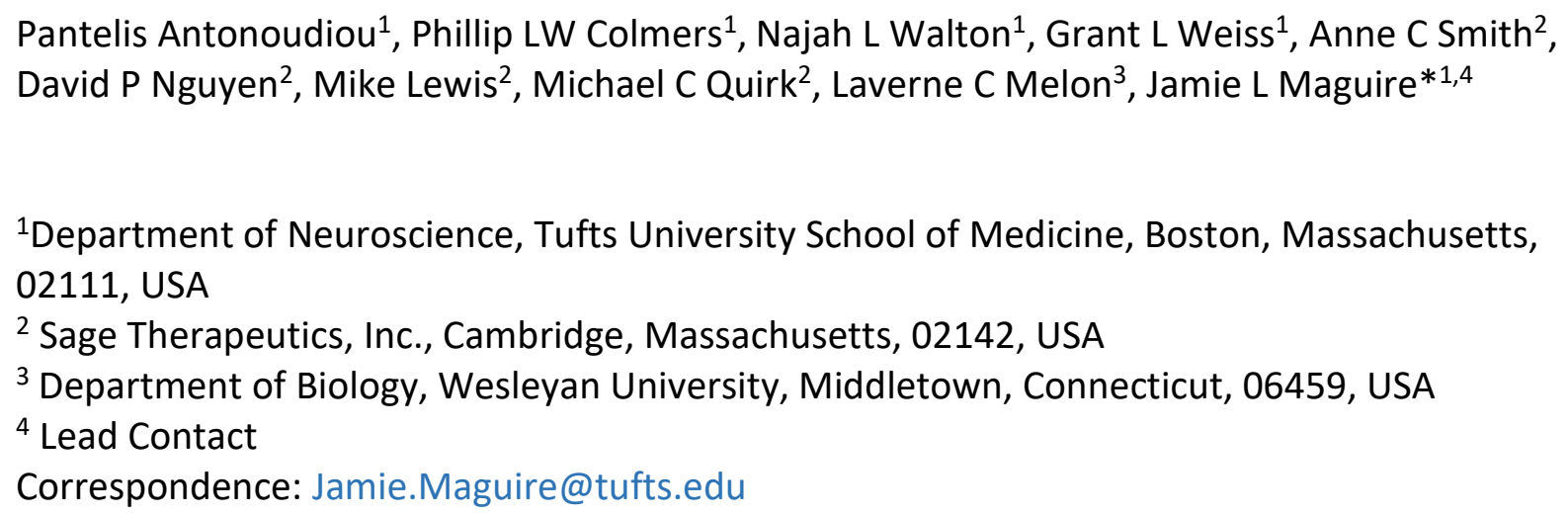




\section{Abstract}

27 Brexanolone (allopregnanolone), was recently approved by the FDA for the treatment of post-partum 28 depression, demonstrating long-lasting antidepressant effects. Despite our understanding of the 29 mechanism of action of neurosteroids as positive allosteric modulators (PAMs) of GABA $A_{A}$ receptors, we 30 still do not fully understand how allopregnanolone exerts these persistent antidepressant effects. Here, 31 we demonstrate that allopregnanolone and similar synthetic neuroactive steroid analogs, SGE-516 (toolcompound) and zuranolone (SAGE-217, investigational-compound), are capable of modulating oscillatory states across species, which we propose may contribute to long-lasting changes in behavioral states. We identified a critical role for interneurons in generating oscillations in the basolateral amygdala (BLA) and

35 a role for delta-containing $\mathrm{GABA}_{\mathrm{A}} \mathrm{Rs}$ in mediating the ability of neurosteroids to modulate network and behavioral states. Actions of allopregnanolone in the BLA is sufficient to alter behavioral states and enhance BLA high-theta oscillations $(6-12 \mathrm{~Hz})$ through delta-containing $\mathrm{GABA}_{\mathrm{A}}$ receptors, a mechanism distinct from other $\mathrm{GABA}_{A}$ PAMs, such as benzodiazepines. Moreover, treatment with the allopregnanolone analog SGE-516 induces long-lasting protection from chronic stress-induced disruption effects of neuroactive steroids. 
48

\section{Introduction}

New antidepressant treatments with proposed novel mechanisms of action, brexanolone and esketamine, recently received FDA approval for the treatment of postpartum- and treatment-resistant depression, respectively. Both of these treatments exert rapid and prolonged antidepressant effects which are not well explained by the proposed mechanisms of action of these compounds, offering the opportunity to explore potential novel mechanisms mediating these sustained antidepressant effects(Daly et al. 2018; Meltzer-Brody et al. 2018).

An interconnected network of brain areas including the basolateral amygdala (BLA) and prefrontal cortex (PFC) are critical for emotional processing in the brain(Calhoon and Tye 2015; Tovote, Fadok, and Lüthi 2015). Dysfunction of this network has been implicated in several neuropsychiatric disorders including depression, post-traumatic stress and anxiety(Babaev, Piletti Chatain, and Krueger-Burg 2018; Calhoon and Tye 2015; Fenster et al. 2018; Tovote, Fadok, and Lüthi 2015). Accumulating evidence suggests that rhythmic synchronization of BLA and PFC circuits is required for the behavioral expression of fear and anxiety(Davis et al. 2017; Felix-Ortiz et al. 2016; Karalis et al. 2016; Likhtik et al. 2014; Stujenske et al. 2014). Moreover, distinct oscillatory states in BLA/PFC areas seem to be associated with aversion and safety, the expression of which depends on inhibitory networks(Davis et al. 2017; Likhtik et al. 2014; Ozawa et al. 2020). Here, we investigated the effect of neurosteroids on network activity in amygdalo-cortical regions implicated in mood and its effects on animal behavior. We show that allopregnanolone (and its analogs) alters oscillations in brain regions implicated in mood and promotes healthy network and behavioral states, providing a molecular and cellular mechanistic underpinning of neuroactive steroid-mediated affective switching(Schiller, Schmidt, and Rubinow 2014). 


\section{Methods and Materials}

71 Methods are described in detail in Supplement 1. EEG recordings were obtained from awake

72 human cortical- and rat frontal-cortical regions. LFP recordings were obtained from the BLA of

73 awake $\mathrm{C57BI} / 6 \mathrm{~J}$ and global Gabrd $/$ mice in response to administration of saline, allo $(10 \mathrm{mg} / \mathrm{kg}$,

74 i.p.), SGE-516 (5 mg/kg, i.p.), and diazepam (1 mg/kg, i.p.). Allopregnanolone-potentiated

75 GABAergic currents were measured in BLA principal and PV+ interneurons using whole cell patch-

76 clamp recording and cell type-specific expression of $\delta$-containing $\mathrm{GABA}_{\mathrm{A}} \mathrm{Rs}$ was examined using

77 immunohistochemistry. Ex-vivo BLA oscillations were recorded in an interface recording chamber, induced by application of $800 \mathrm{nM}$ kainic acid and elevated potassium (7.5 $\mathrm{mM} \mathrm{KCl})$. For ex-vivo optogenetic experiments, a viral vector (AAV8-EF1a-DIO-hChR2(H134R)-mCherry-WPREHGHpA) was delivered in the BLA of PV-cre mice. For optogenetic activation a blue light was delivered through a fiber optic (200 $\mu \mathrm{m}, 0.22 \mathrm{NA}$ ) coupled to a DPSS blue laser (473 nm, $\max$ power $=500 \mathrm{~mW}$, Laserglow technologies). For acute behavioral experiments, C57bl/6j and global $\mathrm{Gabrd}^{-/-}$mice were infused with saline solution $(0.9 \% \mathrm{NaCl})$ or $5 \mu \mathrm{g}$ allo $(2.5 \mu \mathrm{g} / \mu \mathrm{l}$, Tocris). For chronic unpredictable stress (CUS), mice underwent a three-week protocol consisting of daily subjection to alternating stressors. The fMRI (blood-oxygen-level-dependent) BOLD signal was obtained using a Bruker BioSpec 7.0T with a $20-\mathrm{cm}$ horizontal magnet and $20-\mathrm{G} / \mathrm{cm}$ magnetic

87 field gradient quadrature transmit/receive coil (ID 38mm) at the Center for Translational 


\section{Results}

\section{Allopregnanolone analogs alter brain oscillations across species}

93 To examine whether SGE-516 and SAGE-217, with similar molecular pharmacology to

94 brexanolone and allopregnanolone, alter brain states, we recorded cortical EEG from human subjects(Jobert et al. 2012) and rats. Oral application of SAGE-217 in healthy human subjects significantly elevated the power of the delta $(\delta)$, theta $(\theta)$ and beta1 $(\beta 1)$ frequency bands $(\delta$ :

$42.7 \pm 12.30 \%, n=7, p=0.01 ; \theta: 33.9 \pm 9.70 \%, n=7, p=0.01 ; \beta 1: 34.3 \pm 11.80 \%, n=7, p=0.03 ;$ unpaired

t-tests Fig. $1 \mathrm{a}-\mathrm{c})$. Therefore, SAGE-217 seems to produce robust alterations to human brain networks that could promote their anti-depressant effects and serve as a useful readout of target engagement.

We also recorded the cortical EEG from rats treated with SGE-516 and SAGE-217 and quantified the power of oscillations in the low-theta $(2-5 \mathrm{~Hz})$ band (associated with pro-fear states)(Davis et al. 2017; Karalis et al. 2016), high-theta (6-12Hz) band (associated with pro-safety states)(Davis et al. 2017) and beta band (15-30Hz). Similarly, both SGE-516 and SAGE-217 saline $^{6-12 \mathrm{~Hz}}: \quad 50.71 \pm 9.13 \%, \mathrm{n}=27, \mathrm{p}<0.0001$, unpaired t-test; Norm. PowersGE516-saline ${ }^{15-30 \mathrm{~Hz}}$ : $n=20, p<0.0001$, unpaired t-test; Fig. 1d-f). Additionally, SAGE-217 increased the power in the low theta band (Norm. PowersGe516-saline-5Hz: 49.26 $\pm 8.66 \%, n=20, p<0.0001$, unpaired t-test; Fig. 1f). 
111 These results indicate that both allopregnanolone (allo) analogs elevate the power of oscillations

112 consistently across high-theta and beta bands in rats.

Furthermore, we examined the effects of these analogs in mice on the LFP in the

114 basolateral amygdala (BLA), an area implicated in the regulation of mood and anxiety(Babaev,

115 Piletti Chatain, and Krueger-Burg 2018; Calhoon and Tye 2015; Tovote, Fadok, and Lüthi 2015).

116 Acute IP injection of a non-sedative dose of SGE-516 $(5 \mathrm{mg} / \mathrm{kg})$ robustly increased the power in

117 high theta and beta bands (Norm. PowersGe516-saline ${ }^{6-12 \mathrm{~Hz}}$ : 1.58 $\pm 0.35, \mathrm{n}=6, \mathrm{p}<0.0001$; Norm

Power ${ }_{S G E 516-s a l i n e}{ }^{15-30 H z}: 1.70 \pm 0.18, n=6, p<0.0001$, Sidak's multiple comparisons; Fig $\left.1 g-i\right)$. On the

other hand, acute IP injection of SGE-516 in mice lacking $\delta$ subunit GABA $A$ Rs (Gabrd ${ }^{-/-}$; (Mihalek

et al. 1999) altered only the beta band significantly compared to baseline (Norm. PowersGe516-

saline ${ }^{15-30 \mathrm{~Hz}}: 1.25 \pm 0.26, n=5, p=0.0042$; Norm. Power ${ }_{S G E 516-s a l i n e}{ }^{6-12 \mathrm{~Hz}}: 0.80 \pm 0.43, n=5, p=0.06$, Sidak's

122 multiple comparisons; Fig. 1h-i), indicating that the effects of SGE-516 on network states are

123 partly mediated through $G A B A_{A} R \delta$ subunit-containing receptors. These findings suggest that the

124 ability of allo analogs to alter oscillations in the theta and beta range are shared across species.

127 power of the low theta band (Norm. Power diazepam - saline ${ }^{2-5 H z}:-0.28 \pm 0.09, n=5, p=0.03$, Sidak's

128 multiple comparisons; Supplementary Fig. 1a-c) and did not affect the oscillatory power of the

129 high theta band (Norm. Powerdiazepam - saline $6-12 \mathrm{~Hz}$ : $-0.10 \pm 0.06, n=5, p=0.43$, Sidak's multiple 130 comparisons; Supplementary Fig. 1a-c) in BLA. These effects were not different between Wt and $131 \mathrm{Gabrd}^{-/-}$mice $\left(\mathrm{p}^{2-5 \mathrm{~Hz}}>0.99, \quad \mathrm{p}^{6-12 \mathrm{~Hz}}=0.82, \mathrm{p}^{15-30 \mathrm{~Hz}}=0.31, \mathrm{n}_{\mathrm{wt}}=5, \quad \mathrm{n}_{\mathrm{Gabrd}-/-=5}\right.$, Sidak's multiple 132 comparisons; Supplementary Fig. 1c). In addition, a non-sedative dose of diazepam (2.5 mg/kg) 
133 did not affect the oscillatory power of cortical rat EEG in either the low or high theta bands (Norm.

134 Power $_{\text {diazepam - saline }}{ }^{2-5 \mathrm{~Hz}}:-16.66 \pm 19.78 \%, \mathrm{n}=18, \mathrm{p}=0.37$, unpaired t-test; Norm. Power diazepam -saline ${ }^{6-}$

135 12Hz: 9.23 $\pm 11.04 \%, n=18, p=0.47$, unpaired t-test; Supplementary Fig. 1d-f), again demonstrating

136 similarity across species. In both mice and rats the beta-band power was enhanced after

137 diazepam treatment (mouse: Norm. Power diazepam - saline ${ }^{15-30 \mathrm{~Hz}}: 0.29 \pm 0.029, n=5, p=0.0028$, paired

138 t-test; rat: Norm. Power diazepam - saline ${ }^{15-30 H z}$ : 41.00 $\pm 7.81 \%, n=18, p<0.0001$, unpaired t-test;

139 Supplementary Fig. 3c\&f) in agreement with literature reports(Van Lier et al. 2004). These results

140 suggest that neuroactive steroid GABA PAMs confer their effects through distinct network

141 mechanisms from benzodiazepines. 
a

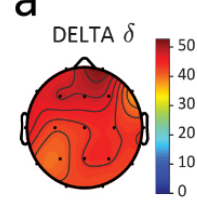

ALPHA $\alpha$

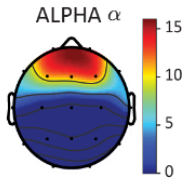

d

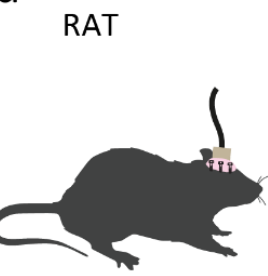

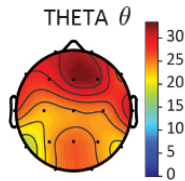

BETA $_{1} \beta_{1}$

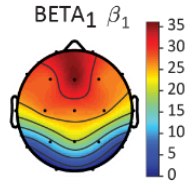

$\mathrm{e}$

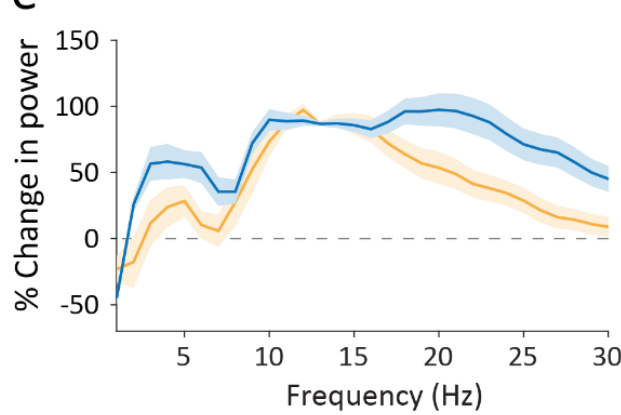

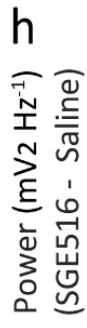

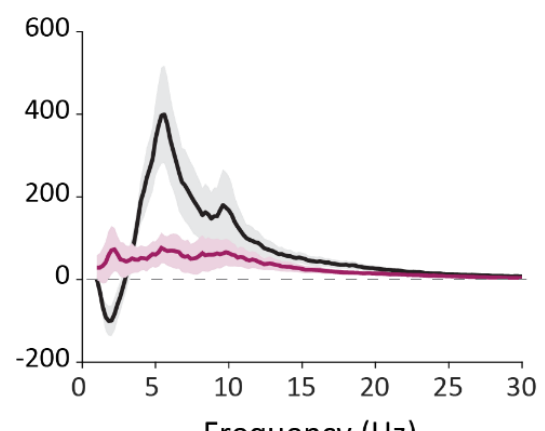

Frequency $(\mathrm{Hz})$
C

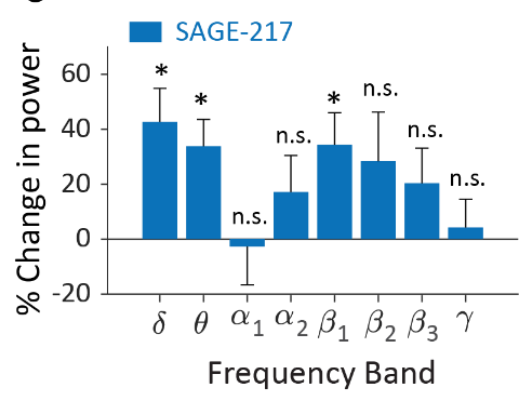

f

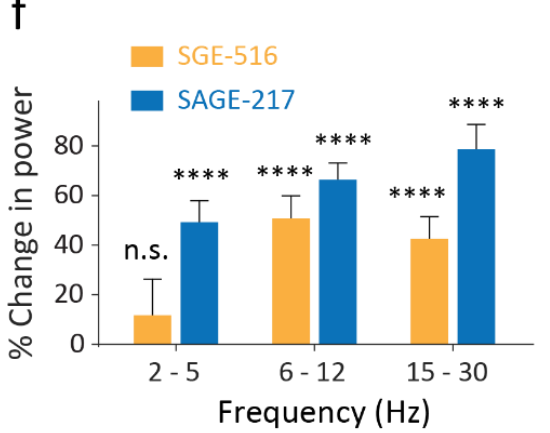

i ${ }_{\text {Gabrd - / }}^{\text {W }}$

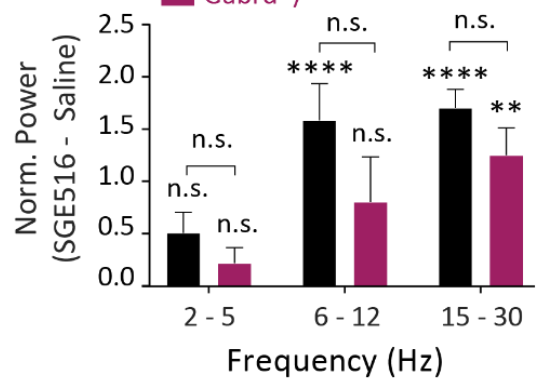

Figure 1. Neuroactive steroids altered brain network dynamics across species. a-c, humans; d-f, rats; g-i, mice. a, Heatmaps organized by frequency band showing regions where percentage change in Human EEG is elevated above vehicle. $\mathbf{b}$, Power spectral mean ( \pm SEM) difference from vehicle in EEG power (defined as percentage change from baseline) with SAGE-217 averaged from frontal electrodes $\mathrm{F3}, \mathrm{Fz}$, and F4. c, Mean ( \pm SEM) change in power from vehicle (defined as percentage change from baseline) for SAGE-217. Shaded regions and error bars represent SEM ( $n=6$ human subjects). $d$, Schematic for cortical EEG recordings in awake rats. e, Power spectral mean difference from vehicle in EEG power (defined as percentage change from baseline) in the rat with SGE-516 (orange) and SAGE217 (blue). $f$, Mean ( \pm SEM) change in power across frequency bands ( $n_{\text {SGE-516 }}=27$ rats, $n_{\text {SAGE-217 }} 20$ rats); stars represent unpaired t-tests. $\mathbf{g}$, Schematic for BLA LFP recordings in awake mice. $\mathbf{h}$, Power spectral density difference between SGE-516 (5 mg/Kg) and saline IP applications. i, Normalized power area difference between acute SGE-516 and saline treatment across multiple frequency bands; Single stars represent paired t-tests between drug treatments. Brackets represent unpaired t-tests between genotypes ( $\mathrm{n}_{\mathrm{wt}}=6$ mice, $\mathrm{n}_{\text {Gabrd }-/=5}=5$ mice). 
a
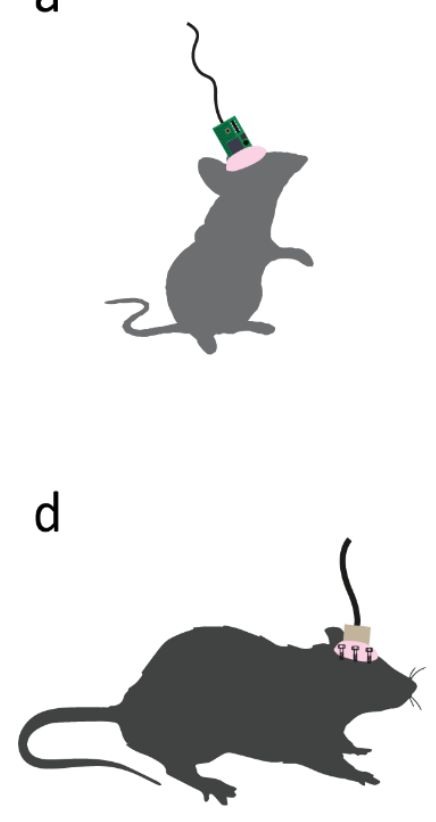

b

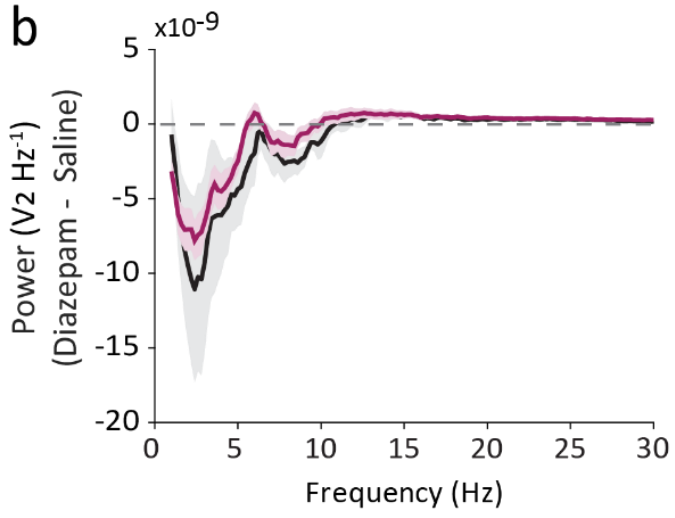

e

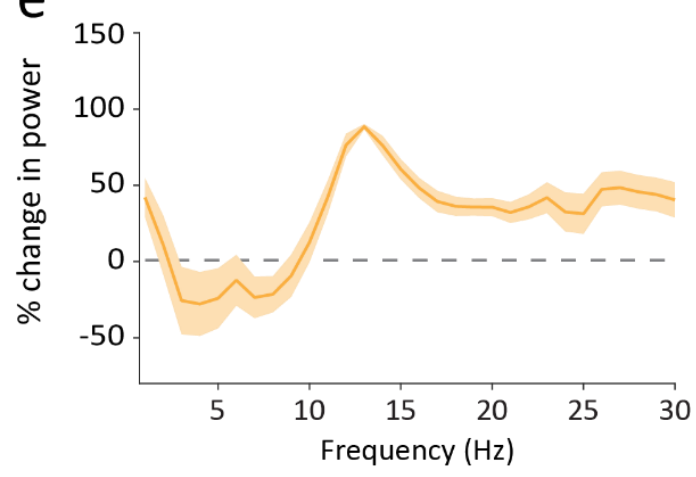

C
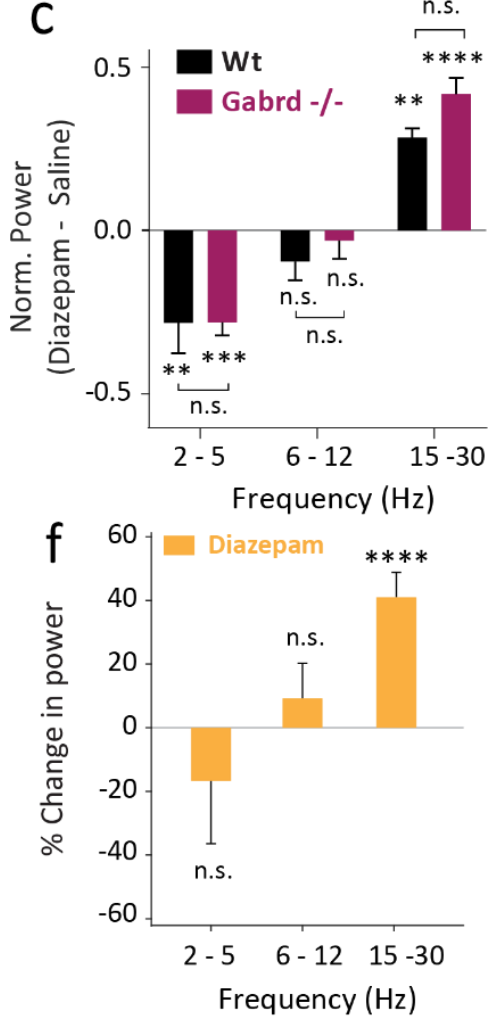

Supplementary Figure 1 (Supporting Figure 1). Diazepam treatment altered theta oscillations differentially from Neurosteroids. a-c, mice; d-f, rats. a, Schematic for BLA LFP recordings in awake mice. b, Power spectral density difference between diazepam $(1 \mathrm{mg} / \mathrm{Kg})$ and saline IP applications. c, Normalized power area difference between acute diazepam and saline treatment across multiple frequency bands; Single stars represent paired t-tests between drug treatments. Brackets represent unpaired t-tests between genotypes ( $\mathrm{n}_{\mathrm{Wt}}=5$ mice, $\mathrm{n}_{\text {Gabrd }} /==5$ mice). $\mathrm{d}$, Schematic for EEG recordings in awake rats. e, Power spectral mean difference from vehicle in EEG power (defined as percentage change from baseline) in the rat with diazepam (orange). $f$, Mean ( \pm SEM) change in power across frequency bands. Shaded regions and error bars represent SEM ( $n_{\text {diazepam }}=18$ rats); stars represent unpaired t-tests. 
153 In order to investigate the effects of acute allo treatment on the BLA network, we recorded the

154 LFP in the BLA of mice (Fig. 2a). Acute IP injection of allo $(10 \mathrm{mg} / \mathrm{kg}$ ) produced robust changes in 155 the pattern of BLA oscillations across multiple frequency bands. Specifically, acute allo 156 administration strongly potentiated the normalized power in the high theta band (Norm. 157 Power $_{\text {allo }}$ - saline $\mathrm{6}^{6-12 \mathrm{~Hz}}: 0.83 \pm 0.117, \mathrm{n}=13, \mathrm{p}<0.0001$, Sidak's multiple comparison, Fig. 2b-e), 158 signature oscillations associated with pro-safety states (Davis et al. 2017), and beta band (Norm. 159 Power allo - saline ${ }^{15-30 H z}: 1.18 \pm 0.117, n=13, p<0.0001$, Sidak's multiple comparison, Fig. 2e). To test whether these effects were mediated through $\mathrm{GABA}_{A} \mathrm{R} \delta$ subunit-containing receptors, which are the predominant site of action for neurosteroids(Lee and Maguire 2014), we repeated the same experiments in $\mathrm{Gabrd}^{-/}$mice. Acute allo application also potentiated the normalized power in the high theta and beta bands (Norm. Power allo - saline ${ }^{6-12 \mathrm{~Hz}}$ : $0.20 \pm 0.054, \mathrm{n}=5, \mathrm{p}=0.02$; Norm. 164 Power allo - saline ${ }^{15-30 H z}: 0.31 \pm 0.068, n=5, p=0.034$, Sidak's multiple comparisons, Fig. $\left.2 b-e\right)$ but to a

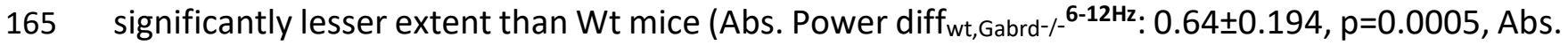
Power diff ${\mathrm{wt}, \mathrm{Gabrd}-\mathrm{-}^{-}}^{15-30 \mathrm{~Hz}}$ : $0.86 \pm 0.196, \mathrm{p}<0.0001, \mathrm{df}=80$, Sidak's multiple comparisons). These

167 findings suggest that acute allo treatment promotes the network communication of pro-safety oscillations in the BLA which are partially attributed to signaling via $G A B A_{A} R \delta$ subunit-containing receptors. 
173 comparisons; Fig. 2e) (Abs. Power diff $_{w t, G a b r d-/-}{ }^{80-120 H z}:-0.003 \pm 0.136, p>0.9999, d f=80$, Sidak's

174 multiple comparison). Therefore, allo reduced the power of BLA oscillations in the high-gamma

175 band which have not previously been attributed to affective behavioral states and are

176 independent of $\mathrm{GABA}_{A} \mathrm{R} \delta$ subunit-containing receptors. The alterations observed in BLA

177 oscillation power cannot be attributed to potentiation or habituation of the second IP injection

178 as the LFP power to repeated saline injection is unchanged in both Wt and $\mathrm{Gabrd}^{-/}$mice (Supp.

179 Figure 2).

To examine the relationship between fast and slow theta-band range oscillations,

182 fear(Davis et al. 2017), we measured the power area ratio of high ${ }^{6-12 \mathrm{~Hz}}$ and low ${ }^{2-5 \mathrm{~Hz}}$ theta bands

183 during IP application of saline and allo $(10 \mathrm{mg} / \mathrm{kg})$. Acute allo application significantly increased

184 the power ratio in comparison to saline injection in Wt mice, indicative of a shift to the signature 185 oscillatory state associated with pro-safety(Davis et al. 2017), but not in Gabrd/- mice (wt:: Norm.

186 Power allo - saline ${ }^{6-12 / 2-5 \mathrm{~Hz}}: 0.64 \pm 0.133, \mathrm{n}=13, \mathrm{p}<0.0001 ; \mathrm{Gabrd}^{-/-}::$Norm. Power allo - saline $^{6-12 / 2-5 \mathrm{~Hz}}$ : -

$1870.06 \pm 0.281, n=5, p=0.91$, Sidak's multiple comparison; Fig. 2f). Therefore, allo administration can 188 switch BLA oscillations to the pro-safety state, an effect which seems to require $G_{A B A} R \delta$ 189 subunit-containing receptors. 
a
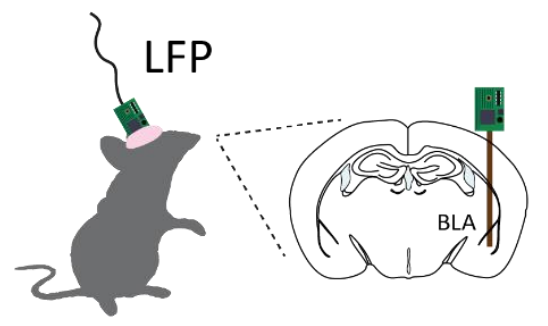

b
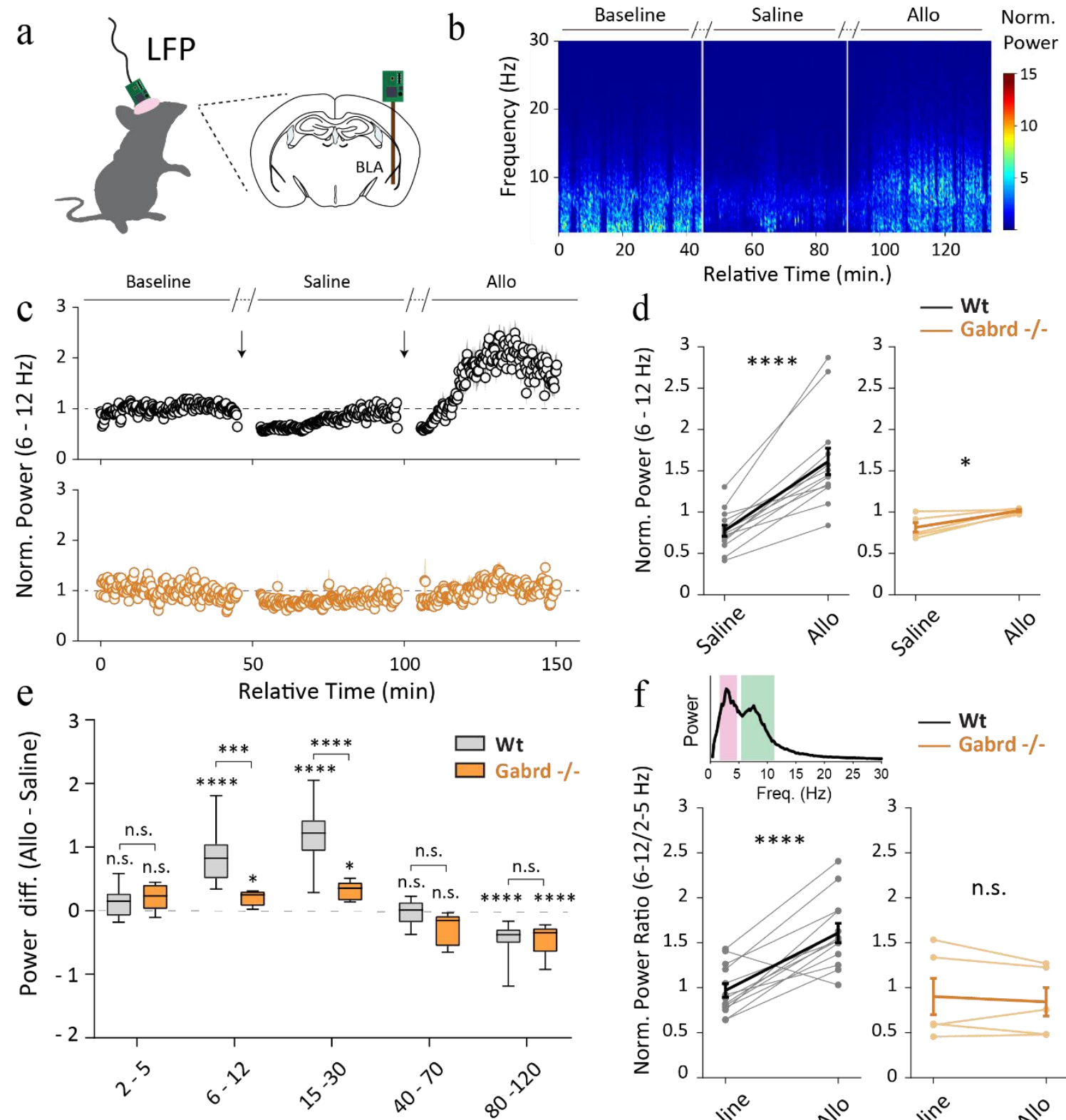

Frequency $(\mathrm{Hz})$
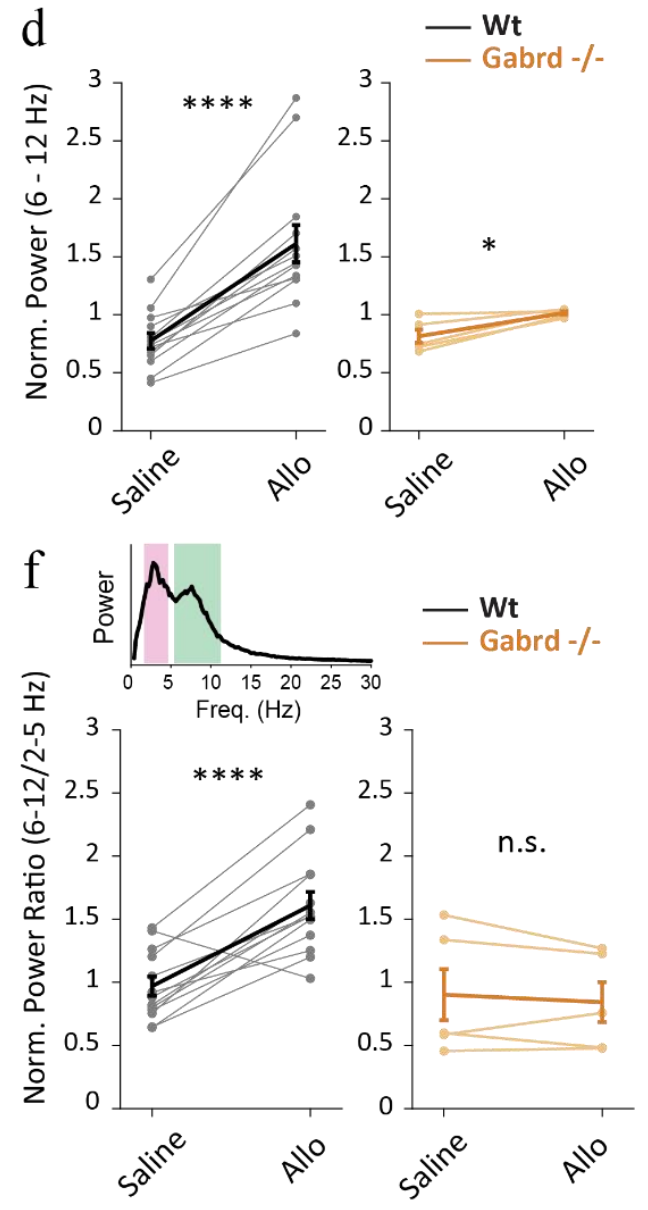

Figure 2. Acute IP application of allopregnanolone altered BLA network oscillations in freely moving mice partly through $\mathrm{GABA}_{\mathrm{A}} \mathrm{R} \delta$ subunit-containing receptors. a, Schematic for LFP recordings in awake mice. $\mathbf{b}$, Representative spectrogram of BLA oscillations during IP application of saline and allo (10 mg/Kg). Normalized power ${ }^{6-12} \mathrm{~Hz}$ is higher during acute application of allo compared to saline treatment. c, Power area $^{6-12 \mathrm{~Hz}}$ normalized to baseline during saline and allo acute treatment across time $\mathbf{d}$, Average dot plot; light color lines indicate individual experiments, dark lines the average and error bars the SEM. e, Normalized power area difference between acute allo and saline treatment across multiple frequency bands; Paired t-tests. f, High(green)/Low(pink) theta power ratio in Wt and Gabrd ${ }^{-/-}$mice. $\mathrm{n}_{\mathrm{wt}}=13$ animals, $\mathrm{n}_{\text {Gabrd } /} /=5$ animals. 

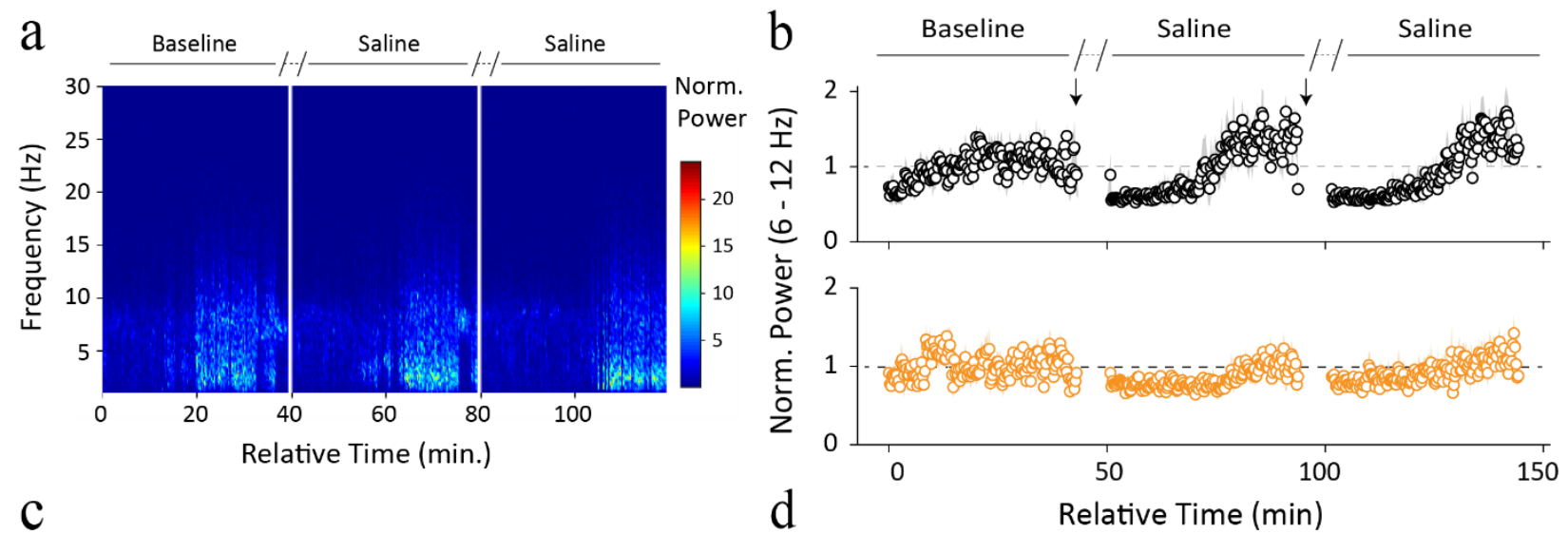

C
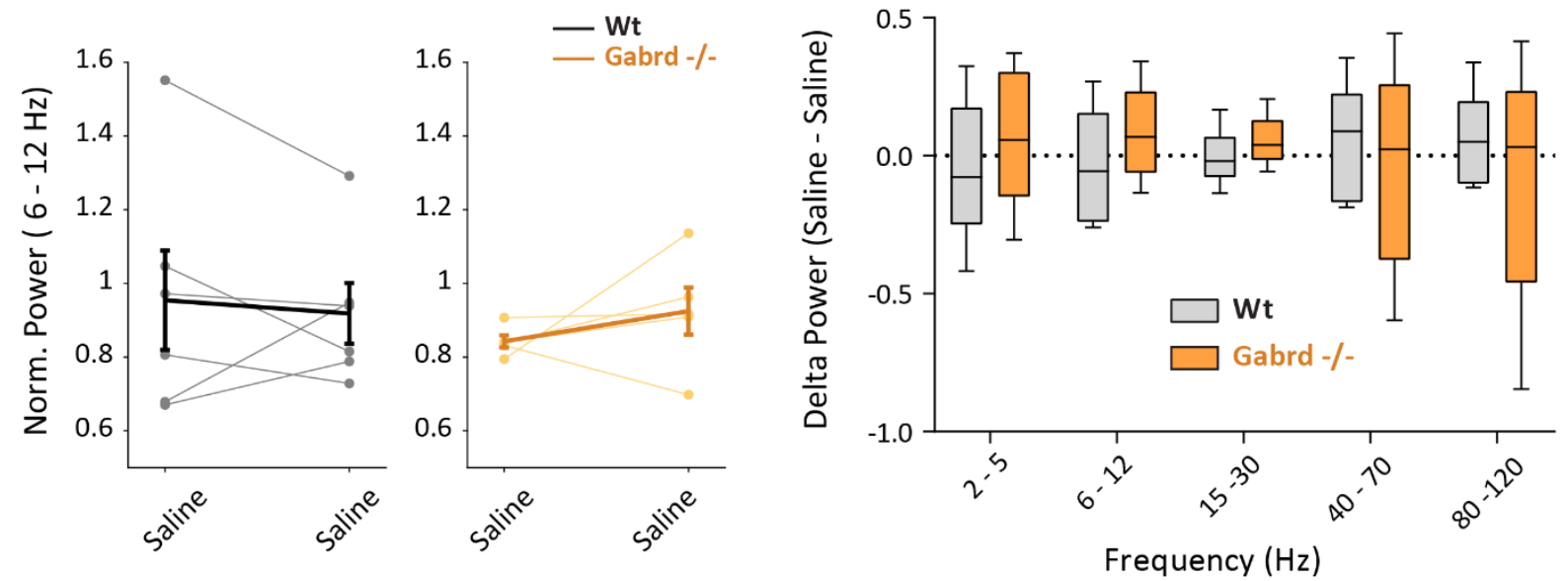

Supplementary Figure 2 (Supporting Figure 2). Repeated IP saline application did not alter BLA network oscillations oscillation. a, Representative spectrogram of BLA oscillations during repeated IP saline application. Power area ${ }^{6-12} \mathbf{H z}$ normalized to baseline during repeated IP saline application $\mathbf{b}$, Across time c, Average dot plot; light color lines indicate individual experiments, dark lines the average and error bars the SEM. d, Normalized power area difference between repeated saline application across multiple frequency bands. Two-way repeated measures ANOVA was used to assess the interactions between frequency bands and repeated saline injections. Wt: $F(4,25)=0.45, p=0.77$; Gabrd $\mathrm{F}(4,20)=0.30, \mathrm{p}=0.87) . \mathrm{n}_{\mathrm{Wt}}=6$ animals, $\mathrm{n}_{\text {Gabrd }-/}=5$ animals. 


\section{principal cells.}

Our experiments suggest that allo actions in the BLA modulates network states through $G A B A_{A} R$

$\delta$ subunit-containing receptors. Given the importance of $\mathrm{PV}^{+}$interneurons in network coordination in BLA(Bartos, Vida, and Jonas 2007; Davis et al. 2017; Karalis et al. 2016; Veres,

Nagy, and Hájos 2017) and the role of neuroactive steroids in potentiating tonic inhibition(Lee and Maguire 2014; Stell et al. 2003), we examined $G_{A B A} R \delta$ subunit-containing receptor and are mostly absent in mice lacking the $\mathrm{GABA}_{A} \mathrm{R} \delta$ subunit specifically in $\mathrm{PV}^{+}$interneurons (PV-

$\mathrm{Gabrd}^{-/}$mice) (Fig. 3a), as PV-Gabrd ${ }^{-/}$mice displayed a significant reduction in count for $\delta$ -

These data suggest that the expression of $\mathrm{GABA}_{A} \mathrm{R} \delta$ subunit-containing receptors is largely restricted to $\mathrm{PV}^{+}$interneurons in $\mathrm{BLA}$.

To investigate the functional effects of $G_{A B A} R \delta$ subunit-containing receptors, we

219 tonic current in $\mathrm{PV}^{+}$but not in principal cells $\left(\mathrm{PV}^{+}: 21.34 \pm 7.68, \mathrm{n}=9, \mathrm{p}=0.02\right.$, paired t-test; Pyr: - 
bioRxiv preprint doi: https://doi org/10.1101/2021.03.08 434156; this version posted March 9, 2021. The copyright holder for this preprint (which was not certified by peer review) is the author/funder, who has granted bioRxiv a license to display the preprint in perpetuity. It is made available under aCC-BY-NC-ND 4.0 International license.

222 Unpaired t-test with Welch's correction; Fig. 3e). These findings suggest that allo potentiates

223 tonic currents in $\mathrm{BLA} \mathrm{PV}^{+}$interneurons through neurosteroid-sensitive $\mathrm{GABA} \mathrm{A}_{\mathrm{A}} \delta$ subunit-

224 containing receptors, which may contribute to the ability to orchestrate synchrony in the BLA

225 (Pavlov et al. 2014).

226

227

228

229

230

231

232

233

234 
a

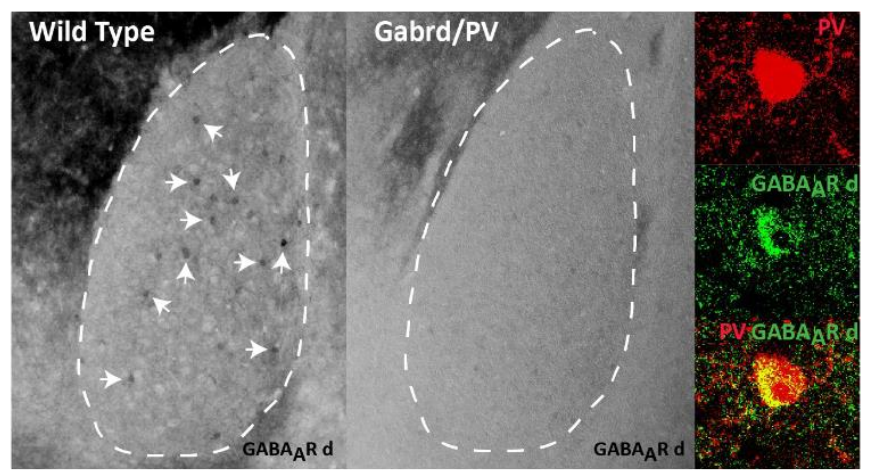

C

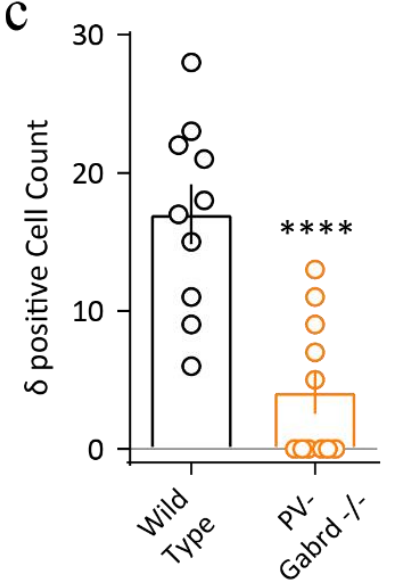

b

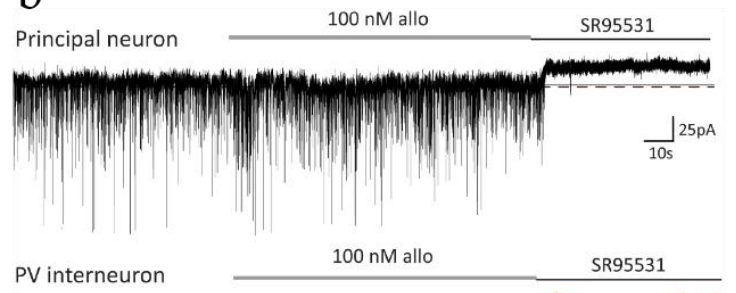

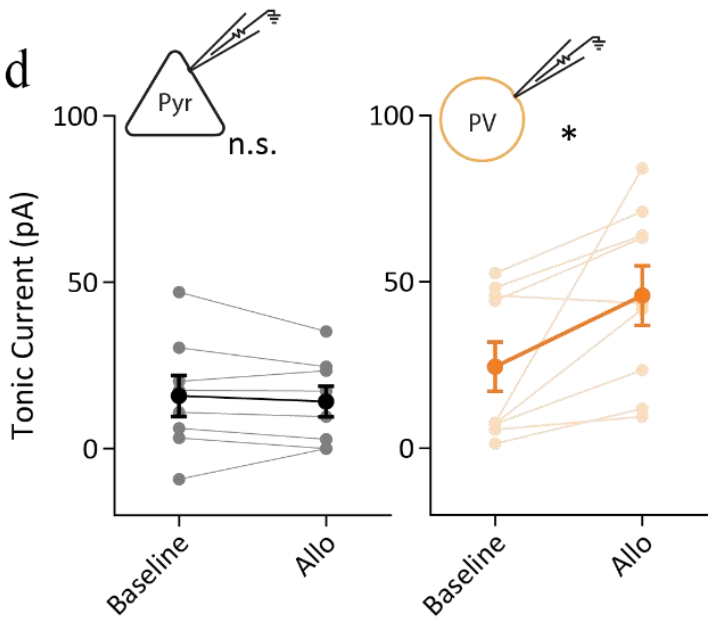

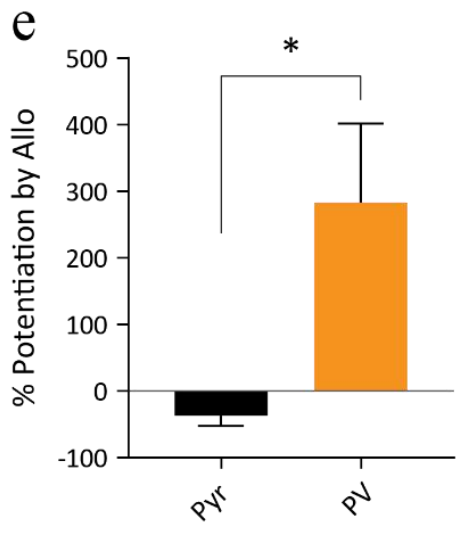

Figure 3. Allopregnanolone enhanced tonic currents in $\mathrm{PV}^{+}$interneurons but not in principal cells of the BLA. a, $\mathrm{PV}^{+}$interneurons highly express $\mathrm{GABA}_{A} \mathrm{R} \delta$ subunit-containing receptors and immunoreactivity for these receptors is absent in PV-Gabrd ${ }^{-/}$mice. $\mathbf{b}$, Representative traces from intracellular recordings in a principal and $\mathrm{PV}^{+}$neurons during $100 \mathrm{nM}$ allo application and block by SR95531 (Gabazine). c, PV-Gabrd ${ }^{-/-}$mice displayed a significant reduction in $\delta$ immunoreactive cells in the basolateral amygdala when compared with controls (Unpaired t-test, $t=4.921$, wt: $n=10$ slices ( 3 mice), $\mathrm{PV}-\mathrm{Gabrd}^{-/}: \mathrm{n}=11$ slices (4 mice)). $\mathbf{d}$, Tonic current in principal cells (pyr) and $\mathrm{PV}^{+}$interneurons (PV) during baseline and $100 \mathrm{nM}$ allo application; paired t-test. e, Percentage potentiation of tonic current between allo and baseline application; Unpaired t-test with Welch's correction. $\mathrm{n}_{\mathrm{PV}}=9$ cells, $\mathrm{n}_{\text {Pyr }}=10$ cells. 
240 Isolated BLA networks can generate local brain oscillations

241 In order to examine the importance of $\mathrm{PV}^{+}$interneurons in controlling the BLA circuit we sought

242 to establish an ex-vivo oscillation model in isolated BLA networks of mice. Under regular aCSF

243 conditions the LFP was silent (Supplementary Fig. 3a-b). However, addition of kainic acid (800

$244 \mathrm{nM}$ ) and elevated $\mathrm{KCl}\left(\mathrm{High} \mathrm{K}^{+} / \mathrm{KA}\right)$ into regular aCSF induced robust network oscillations at

245 gamma ( $\gamma$ )-band frequency centered at $40 \mathrm{~Hz}$ (Fig. 4a-c; Supplementary Fig. 3a-b). The current

246 generator of these oscillations was situated within the BLA circuit, as nearby regions did not

247 exhibit any oscillations (Supplementary Fig. 3c) and the hippocampus was removed to eliminate

248 hippocampal volume conduction. Therefore, the isolated mouse BLA network is capable of

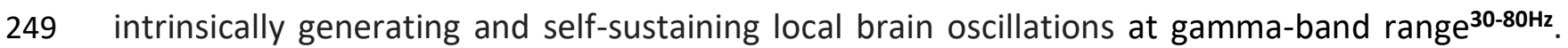

250 Furthermore, the generation of these oscillations does not seem to require phasic synaptic

251 excitation as a selective AMPAR antagonist (Gyki 53655, n=7 slices; 6 at $20 \mu \mathrm{M}, 1$ at $10 \mu \mathrm{M}$ ) did

252 not significantly alter gamma oscillation peak power $(2.14 \pm 0.57$ of baseline, one-sample t-test:

$253 \mathrm{p}=0.09, \mathrm{n}=7$ slices (4 mice); Fig. 4d-e). On the other hand, gabazine application (10 $\mu \mathrm{M})$ strongly

254 suppressed gamma oscillation peak power $(0.17 \pm 0.033$ of baseline, one-sample t-test: $p<0.0001$,

$255 \mathrm{n}=8$ slices (4 mice); Fig. 4f-g) indicating that fast synaptic inhibition is critical for gamma oscillation

256 emergence in BLA. Interestingly, following the collapse of gamma oscillations, rhythmic activity

257 in the theta $(\theta)$ frequency range ${ }^{3-12 \mathrm{~Hz}}$ emerged (368.52 \pm 165.61 of baseline, $\mathrm{n}=9$ slices (4 mice);

258 Fig. 4f-g). In 4 out of 9 of these experiments additional low-frequency events emerged

259 (Supplementary Fig. 3e-f). Therefore, isolated BLA circuits are capable of generating network 

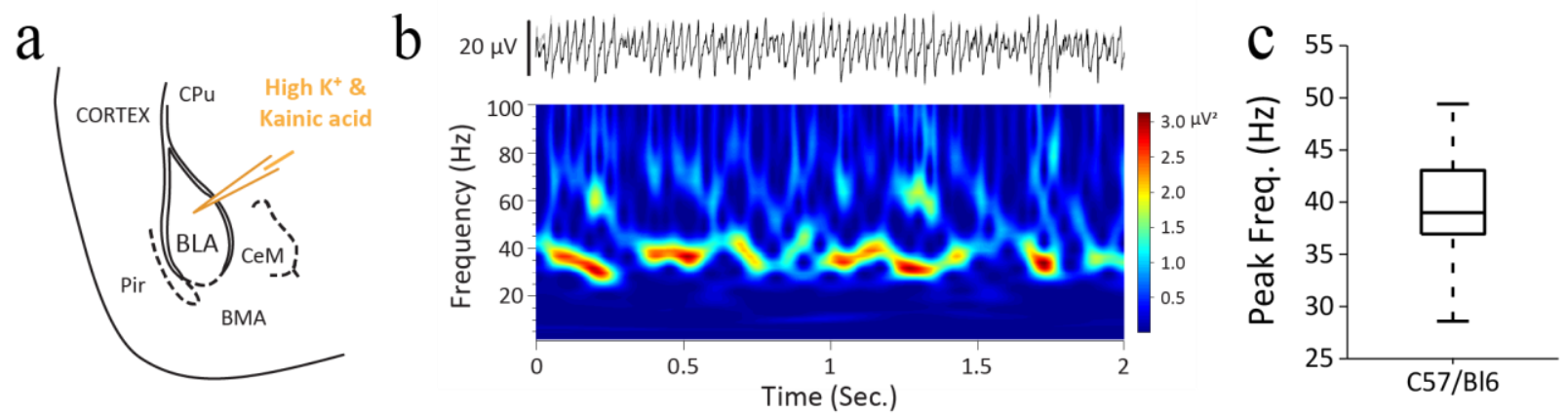

d

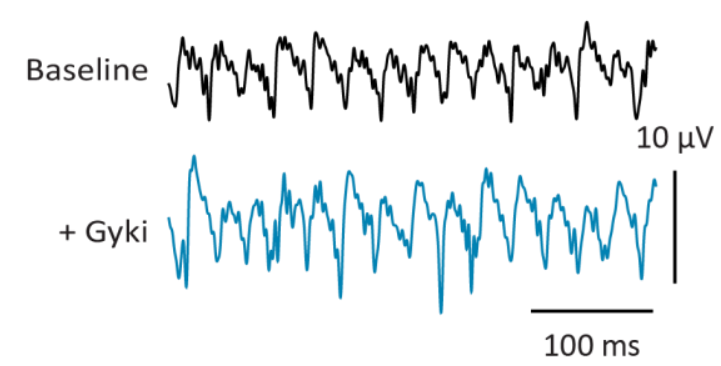

e

Gyki 53655

f

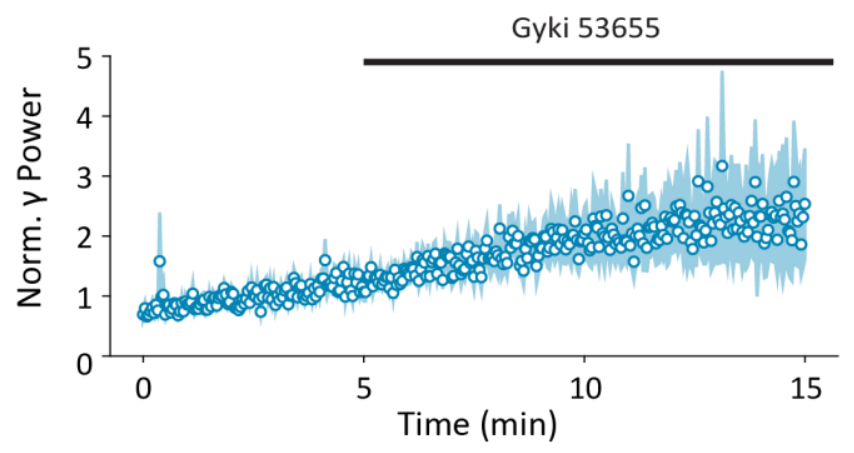

$\mathrm{g}$

Baseline

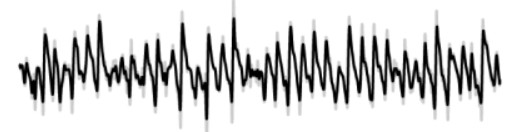

$+\mathrm{Gbz}$

(7 min.)

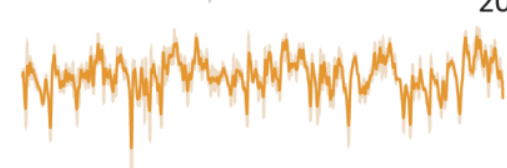

$20 \mathrm{uV}$

$+\mathrm{Gbz}$

(10 $\mathrm{min})$.

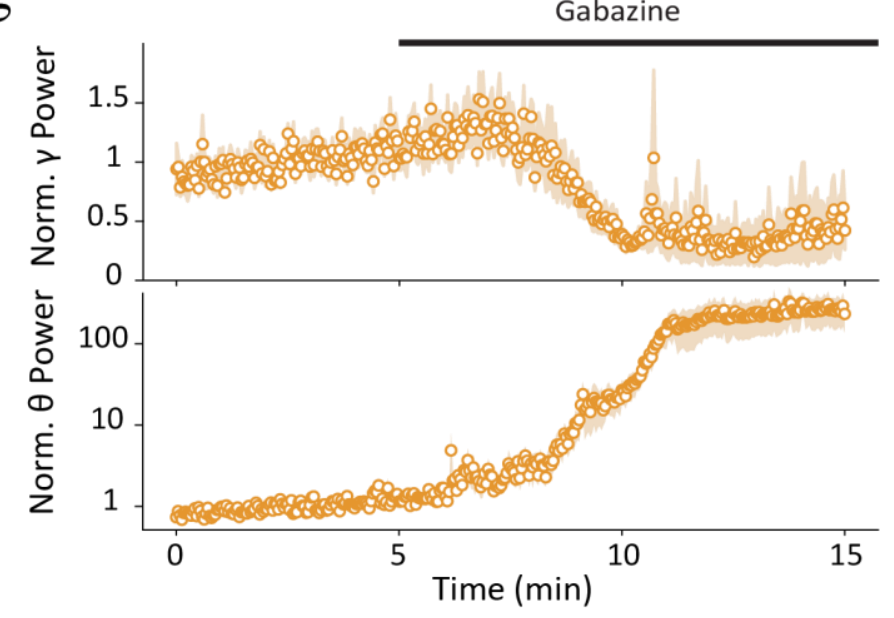

Figure 4: Isolated mouse BLA networks ex-vivo generated brain oscillations in the gamma and theta band-range. a, Schematic illustration of LFP setup for recording of ex-vivo gamma $(\gamma)$ oscillations in mouse BLA slices. All experiments were performed in the presence of High $\mathrm{K}^{+} / \mathrm{KA}$ solution. $\mathbf{b}$, top: Representative gamma oscillation LFP trace from BLA and bottom: Wavelet transformation. c, Peak frequency box plot of BLA gamma oscillations ( $n=42$ slices). d-e, Gyki 53655 application to BLA gamma oscillations. d, Representative LFP traces during baseline (black) and Gyki 53655 application (blue). e, Normalized gamma peak power; dots represent mean and shaded region represents SEM ( $n=7$ slices; 6 at $20 \mu \mathrm{M}, 1$ at $10 \mu \mathrm{M})$. f-g, Gabazine application to BLA gamma oscillations. f, Representative LFP traces during baseline (black) and gabazine application (orange). g, Normalized gamma (top) and theta (bottom) peak power change; dots represent mean and shaded region represents SEM ( $n=9$ slices at 10 $\mu \mathrm{M})$. 
a
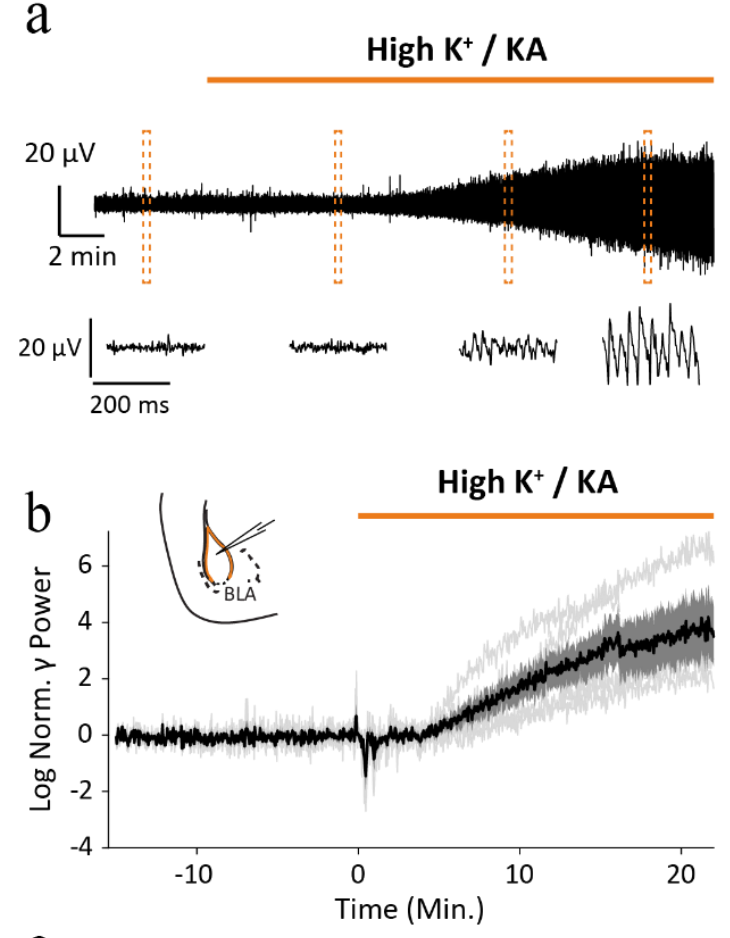

e

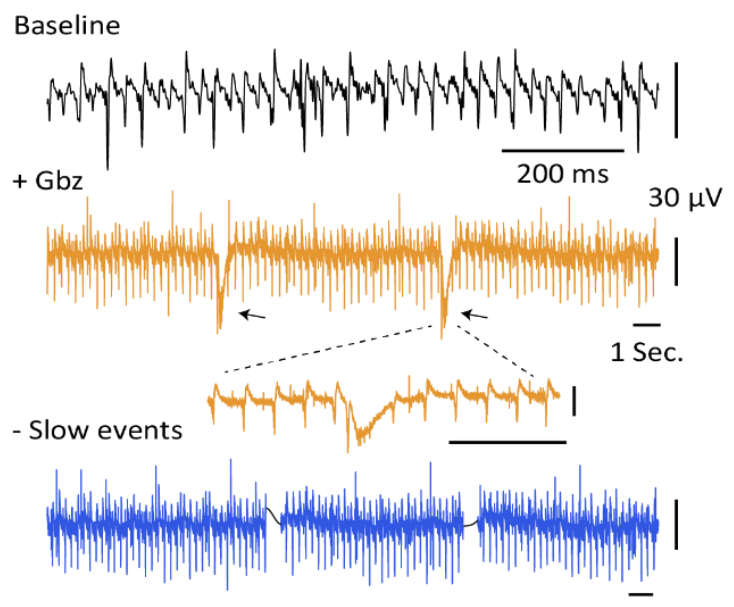

C

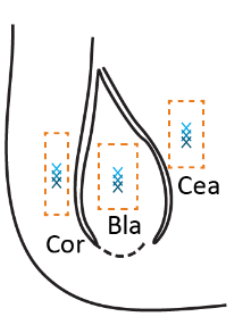

Cor Bla Cea

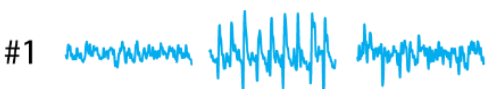

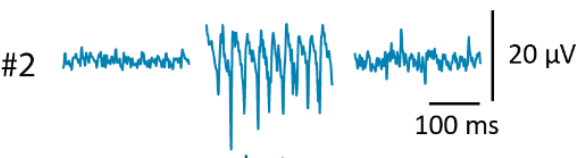

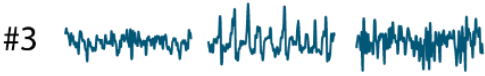

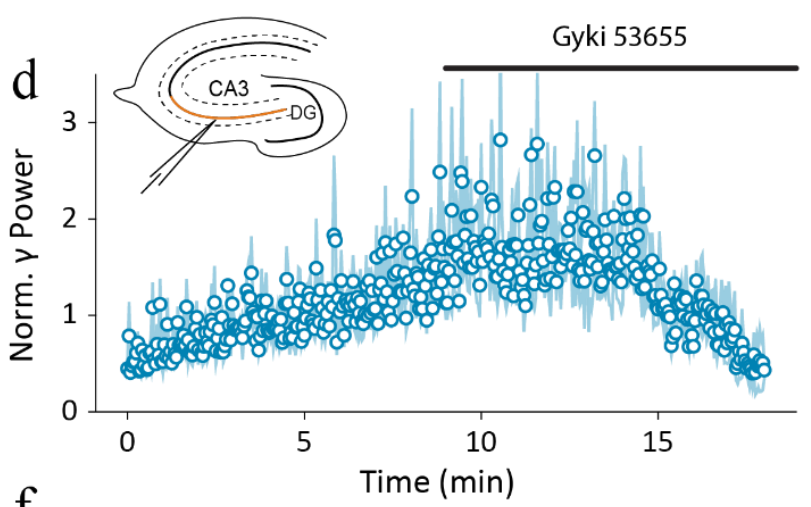

f

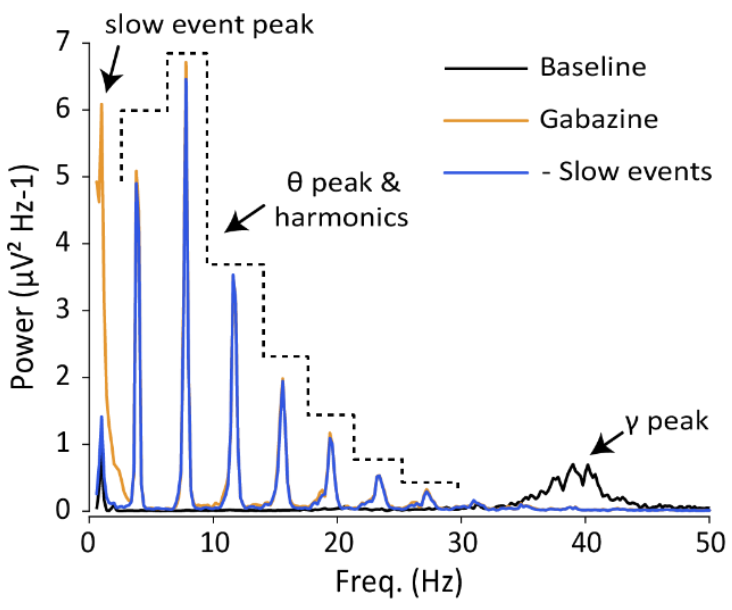

Supplementary Figure 3 (Supporting Figure 4). a-b, Application of kainic acid (800 nM) in aCSF with elevated $\mathrm{KCl}$ (7.5 mM) induces gamma oscillations. a, Top- Representative LFP trace and BottomMagnified traces from orange rectangles. $\mathbf{b}$, Normalized gamma peak power ${ }^{\mathbf{3 0}-80 \mathrm{~Hz}}$. Grey traces represent individual experiments ( $n=9$ slices), black trace the mean and shaded area the SEM. c, Gamma oscillations can only be recorded in BLA and not surrounding areas; Left- schematic of approximate LFP recording positions in Right- rows represent LFP recordings from separate slices ( $n=3$ slices). d, Gyki 53655 application (20 $\mu \mathrm{M})$ suppresses carbachol-induced (5 $\mu \mathrm{M})$ oscillations in CA3; dots represent mean and shaded region represents SEM. e-f, Induction of additional slow events from gabazine application in BLA. e, Representative LFP recordings from BLA and $\mathbf{f}$, respective power spectral density. 
$266 \mathrm{PV}^{+}$interneurons can synchronize and control the BLA network

267 To assess whether fast-synaptic inhibition is sufficient to entrain the local BLA network and control oscillatory states we employed optogenetics to recruit BLA inhibitory interneurons. We targeted $\mathrm{PV}^{+}$interneurons, which are the major class of GABAergic cells in BLA and have been suggested to provide powerful control over the somatic regions of local neurons(Bartos, Vida, 271 and Jonas 2007; Krabbe, Gründemann, and Lüthi 2018; Veres, Nagy, and Hájos 2017). Infusion of 272 AAV-DIO-ChR2-mCherry viral vector into the BLA of PV-cre mice resulted in expression of ChR2mCherry in BLA PV ${ }^{+}$interneurons (Fig. 5a-b). Pulsed blue light $\left(473 \mathrm{~nm}\right.$ ) photo-excitation ${ }^{5-60 \mathrm{~Hz}}$ produced robust phase-locked LFP responses suggesting that synchronized GABAergic release 275 from $\mathrm{PV}^{+}$interneurons can be detected in the BLA LFP (Fig. 5c-d; Supplementary Fig. 4a; $p^{5 \mathrm{~Hz}}=0.0004, p^{10 \mathrm{~Hz}}<0.0001, \mathrm{p}^{20 \mathrm{~Hz}}<0.0001, \mathrm{p}^{30 \mathrm{~Hz}}=0.0001, \mathrm{p}^{40 \mathrm{~Hz}}=0.0006, \mathrm{p}^{50 \mathrm{~Hz}}=0.016, \mathrm{p}^{60 \mathrm{~Hz}}>0.99 ;$

277 Bonferroni's multiple comparisons test). In order to test if $\mathrm{PV}^{+}$interneurons can entrain the BLA

278 network and control BLA oscillatory state ex-vivo we examined the effects of pulsed optoexcitation during KA induced gamma. Pulsed blue light photo-excitation $(5-60 \mathrm{~Hz})$ increased the power ratio (Fig. 5e-f; $\mathrm{p}^{5 \mathrm{~Hz}}=0.094, \quad \mathrm{p}^{10 \mathrm{~Hz}}<0.0001, \quad \mathrm{p}^{20 \mathrm{~Hz}}<0.0001, \quad \mathrm{p}^{30 \mathrm{~Hz}}<0.0001, \quad \mathrm{p}^{40 \mathrm{~Hz}}=0.076$, $40 \mathrm{~Hz}$ reliably entrained the ongoing kainate-induced gamma oscillations (Supplementary Fig. 4binterneurons strongly suppressed ongoing gamma oscillations during light stimulation period 
bioRxiv preprint doi: https://doi org/10.1101/2021.03.08.434156; this version posted March 9 2021. The copyright holder for this preprint (which was not certified by peer review) is the author/funder, who has granted bioRxiv a license to display the preprint in perpetuity. It is made available under aCC-BY-NC-ND 4.0 International license.

$288 p=0.0003, n=70$, Spearman correlation, Fig. 5i), indicating that the stronger the light intensity the

289 larger the gamma power suppression. Moreover, blue light stimulation had no effect in control

290 slices from PV-cre mice that were not injected with the ChR2 viral vector $(0.95 \pm 0.023$ of baseline,

$291 \mathrm{n}=6$ slices, $\mathrm{p}=0.29$, Dunnett's multiple comparison; Supplementary Fig. 4d). Taken together these

292 results suggest that $\mathrm{GABAergic} \mathrm{PV}^{+}$interneurons are powerful regulators of the local network

293 activity and can control oscillatory states in BLA.

294

295 

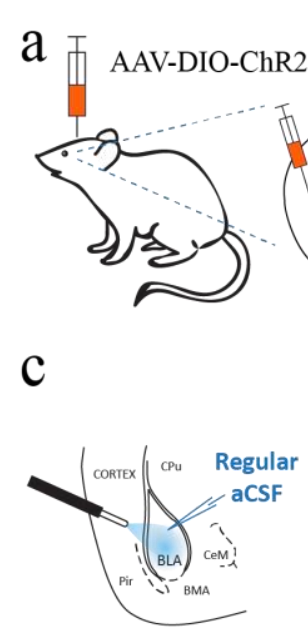

e

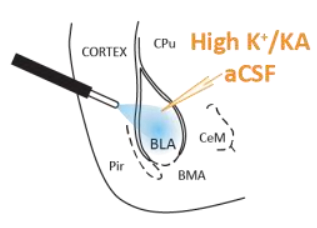

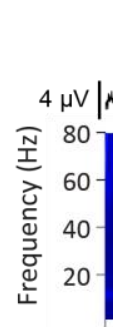

$-1$

$30 \mathrm{~Hz}-$
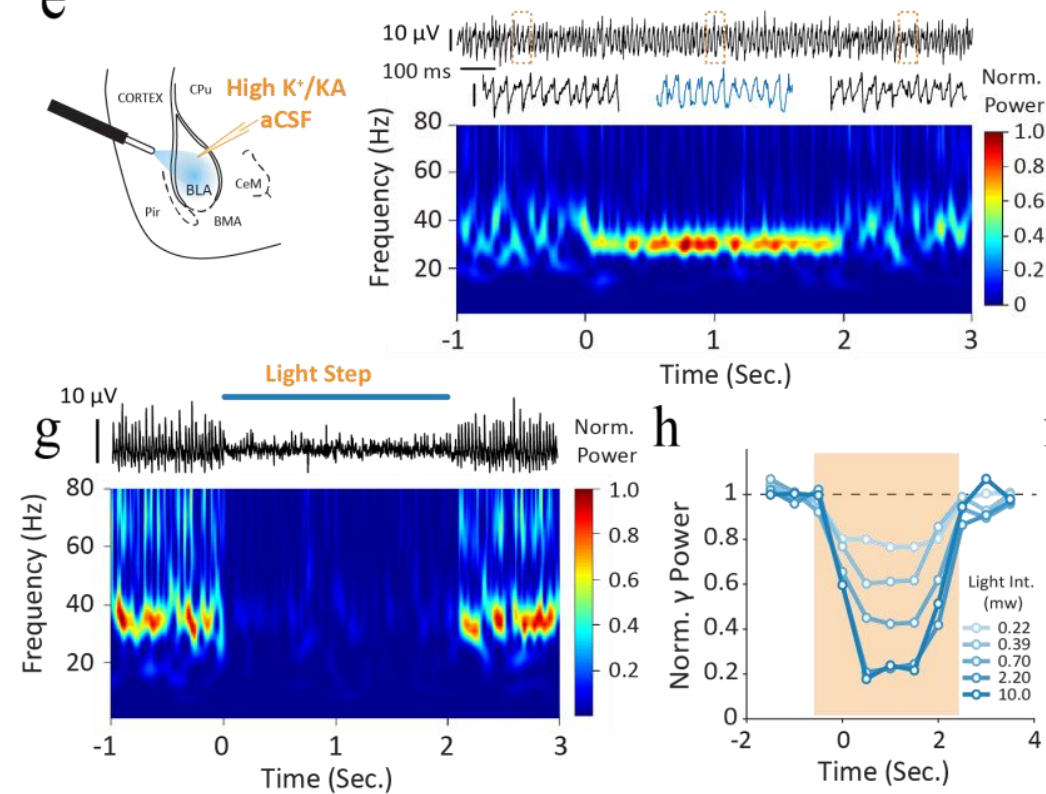

i

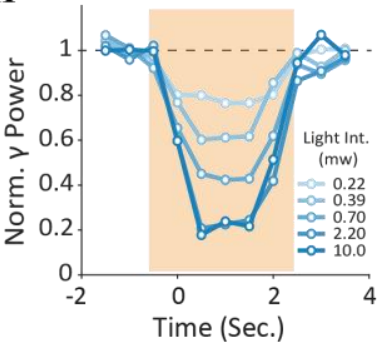

$\mathrm{b}$
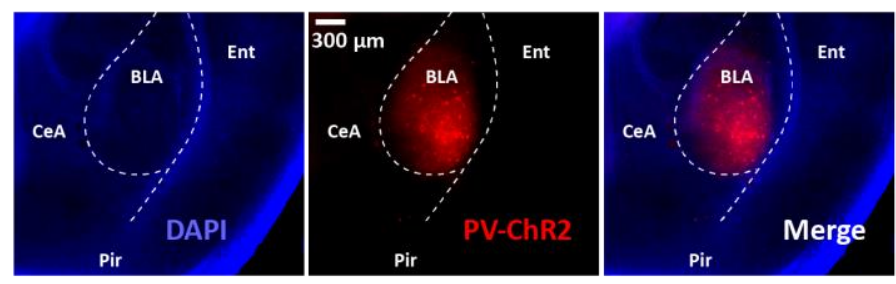

Pir

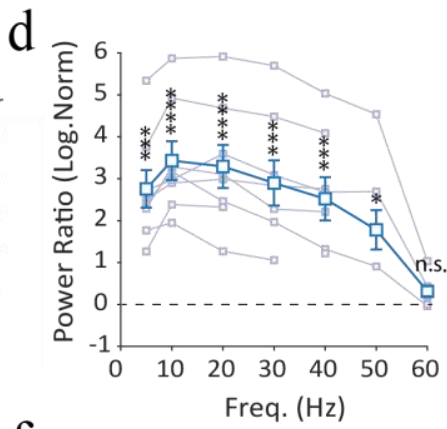

f

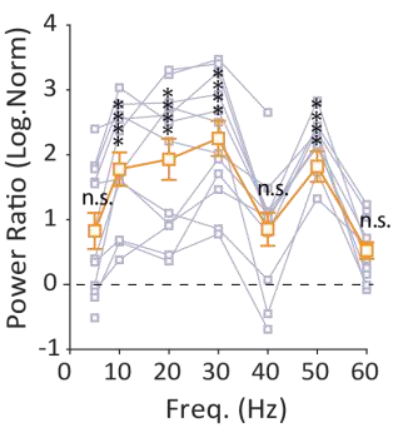

Figure 5. $\mathrm{PV}^{+}$interneurons can synchronize and control the BLA network. a, Schematic of viral infusion of AAV-DIO-ChR2-mCherry in mouse BLA. $\mathbf{b}$, Representative fluorescence images of ChR2-mCherry expression in BLA; $\mathrm{CeA}=$ Central Amygdala, BLA=basolateral amygdala, Pir=Piriform Cortex; Ent=Entorhinal Cortex. c, left- schematic illustration of LFP-opto setup for ChR2 experiments in aCSF; Right- top: Representative gamma oscillation LFP trace from BLA and bottom: Wavelet transformation. $\mathbf{d}$, LFP power ratio between pulsed stimulation and baseline periods ( $n=8$ slices). e-f, same as $c$-d during High $K^{+} / K A$ ( $n=12$ slices) Bonferroni's multiple comparisons test. $\mathbf{g}-\mathbf{i}$, Sustained $\mathrm{PV}^{+}$interneuron excitation suppresses kainateinduced gamma oscillations. g, top: Representative gamma oscillation LFP trace from BLA and bottom: Wavelet transformation. $\mathbf{h}$, Normalized gamma power to baseline across time for different light intensities. i, Gamma power normalized to baseline across light intensities ( $\mathrm{n}=15$ slices). 


\section{a Regular aCSF}
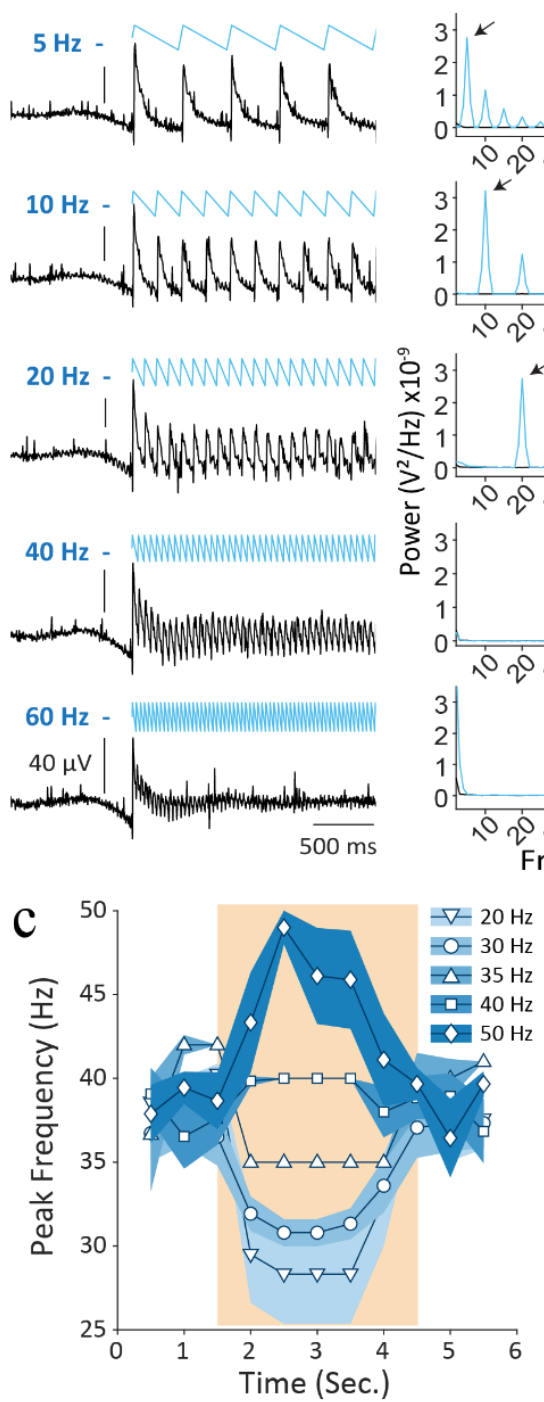

b High $\mathrm{K}^{+} / \mathrm{KA}$ aCSF
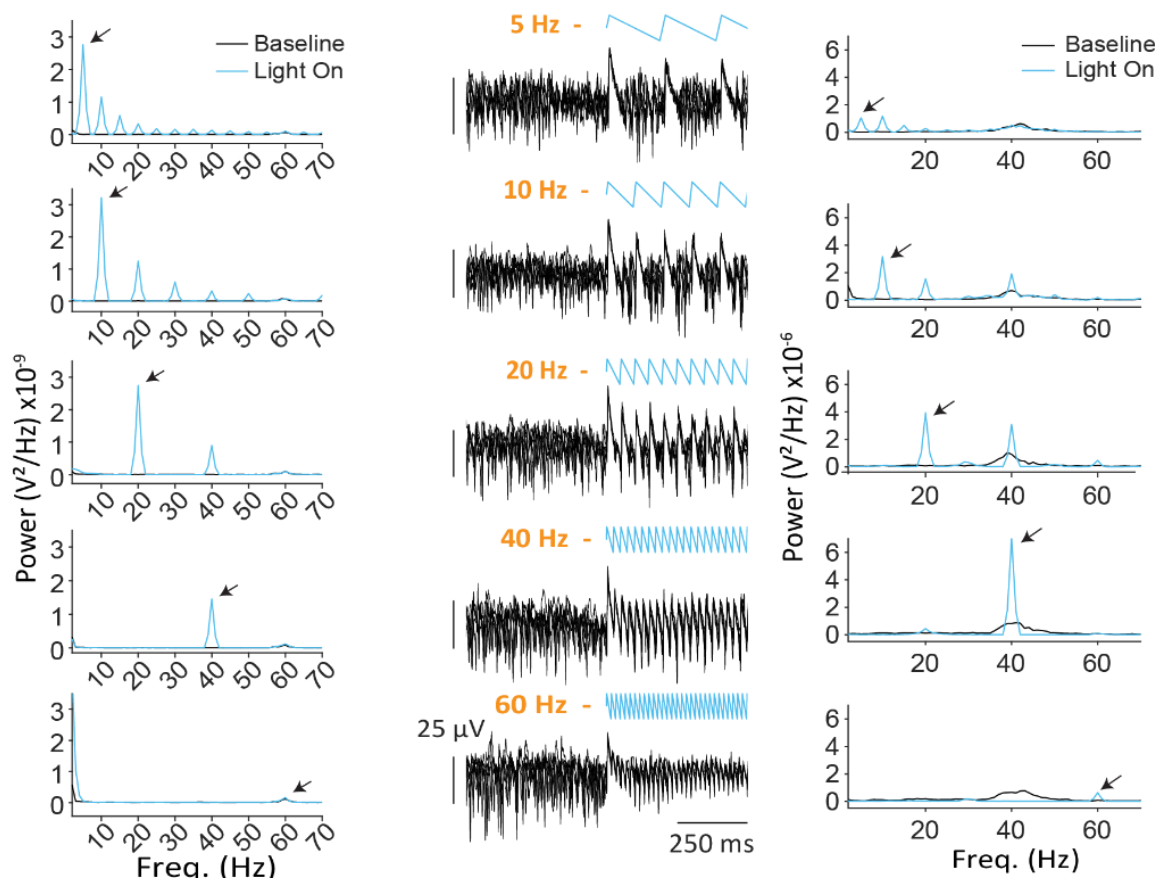

$10 \mathrm{~Hz}$
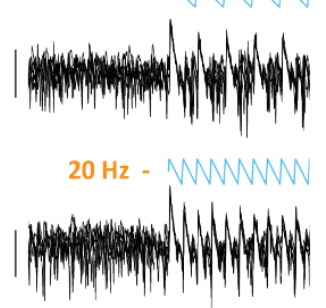

$40 \mathrm{~Hz}-$ MMMMMMMMMMN
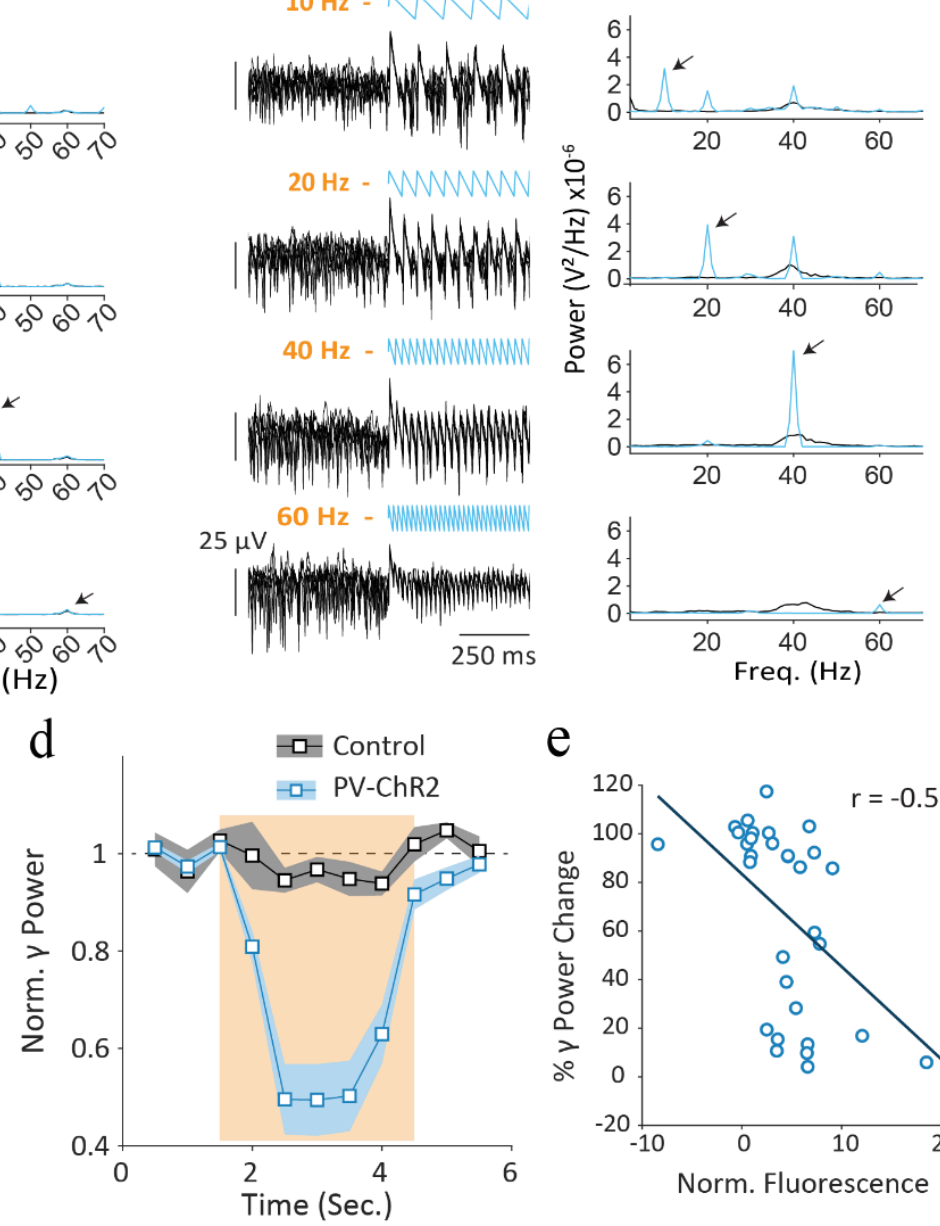

e

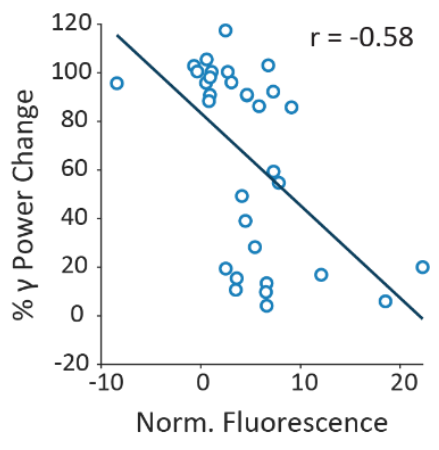

Supplementary Figure 4 (Supporting Figure 5). a-b, Left- Representative LFP recordings from BLA and Right- associated PSDs obtained during $\mathrm{PV}^{+}$interneuron pulsed photo-excitation (black arrows indicate peaks at stimulation frequency). c, Average Peak frequency of oscillations during $\mathrm{PV}^{+}$interneuron pulsed photo-excitation, shaded region=SEM; $n$ slices: $20 \mathrm{~Hz}=12,30 \mathrm{~Hz}=15,35 \mathrm{~Hz}=3,40 \mathrm{~Hz}=13,50 \mathrm{~Hz}=9$. d, Average Normalized gamma power to baseline in controls ( $n=6$ slices) and PV-ChR2 animals during sustained photo-excitation, shaded region=SEM ( $n=23$ slices). e, Gamma Power change during sustained $\mathrm{PV}^{+}$interneuron photo-excitation plotted against normalized BLA fluorescence $(r=-0.58$, $\mathrm{p}=0.0005, \mathrm{n}=32$ slices, Spearman Correlation). Light intensity at $10 \mathrm{~mW}$. 
301 Acute allopregnanolone infusion into the BLA alters behavioral states mediated

In order to determine whether allo can exert effects in the BLA to alter avoidance behaviors, we number of entries) in the open field center when compared with vehicle controls (Fig. 6a-c; time with vehicle controls (Fig. 6d-e \& g-h). The number of entries made in the lit area of the lightdark box and in the open arm of the elevated plus maze did not differ between Wt mice that were infused with allo or vehicle (Fig. $6 f \&$ i; time in lit area allo-veh: $1.55 \pm 4.68, n_{\text {veh }}=10, n_{\text {allo }}=13$, $p=0.75$, unpaired $t$-test; time in open armallo-veh: $-0.32 \pm 4.24, n_{\text {veh }}=11, n_{\text {allo }}=12, p=0.94$, unpaired $t-$ in Wt mice, as indicated by the increased time they spend in anxiogenic regions of the behavioral apparatus. Further, mice treated with allo had a robust reduction in the amount of time spent immobile in the tail suspension test compared to vehicle treated controls (Fig. 6j-k; time immobileallo-veh: $-79.29 \pm 11.15, n_{\text {veh }}=12, n_{\text {allo }}=11, p<0.0001$, unpaired $t$-test). These effects cannot 
323 mice was not different in the open field and light dark box (Supplementary Fig. 5b-c; open-fieldallo-

324 veh: $-67.73 \pm 292.2 \mathrm{~s}, \mathrm{n}_{\text {veh }}=11, \mathrm{n}_{\text {allo }}=11, \mathrm{p}=0.82$, unpaired t-test; time in lit area allo-veh $105 \pm 227.4 \mathrm{~s}$,

$325 \mathrm{n}_{\text {veh }}=11, \mathrm{n}_{\text {allo }}=13, \mathrm{p}=0.65$, unpaired $\mathrm{t}$-test). Importantly, these data demonstrate robust

326 behavioral impacts of acute allo infusion restricted only on the BLA network.

To assess whether delta $(\delta)$ containing GABA receptors might play a role we repeated the

328 same battery of test in $\mathrm{Gabrd}^{-/-}$mice. Acute allo infusion in $\mathrm{Gabrd}^{-/}$mice $(5 \mu \mathrm{g})$ did not change

329 the time spent in the lit area of the light dark box (Fig. 6e; time in lit area allo-veh: $0.56 \pm 35.15 \mathrm{~s}$,

$330 \mathrm{n}_{\text {veh }}=9, \mathrm{n}_{\text {allo }}=11, \mathrm{p}=0.99$, unpaired t-test), open arm of the elevated plus maze (Fig. $6 \mathrm{~h}$; time in

331 open armallo-veh: $-27.67 \pm 25.29 \mathrm{~s}, \mathrm{n}_{\text {veh }}=9, \mathrm{n}_{\text {allo }}=11, \mathrm{p}=0.29$, unpaired t-test) or time immobile in the

332 tail suspension test (Fig. $6 \mathrm{k}$; time immobileallo-veh: $-19.75 \pm 18.54 \mathrm{~s}, \mathrm{n}_{\text {veh }}=8, \mathrm{n}_{\text {allo }}=10, \mathrm{p}=0.30$,

333 unpaired t-test). Therefore, these data suggest that the behavioral effects of acute allo infusion

334 into the $B L A$ requires $G A B A_{A} R \delta$ subunit-containing receptors. 
b

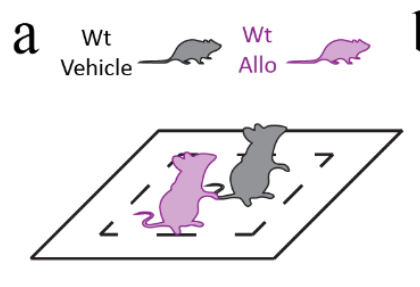

d
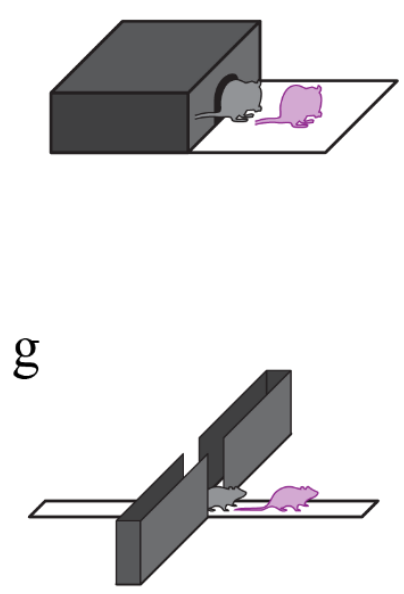

$\mathrm{j}$

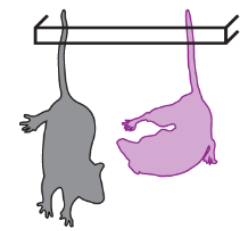

e

$\mathrm{h}$

$\mathrm{k}$
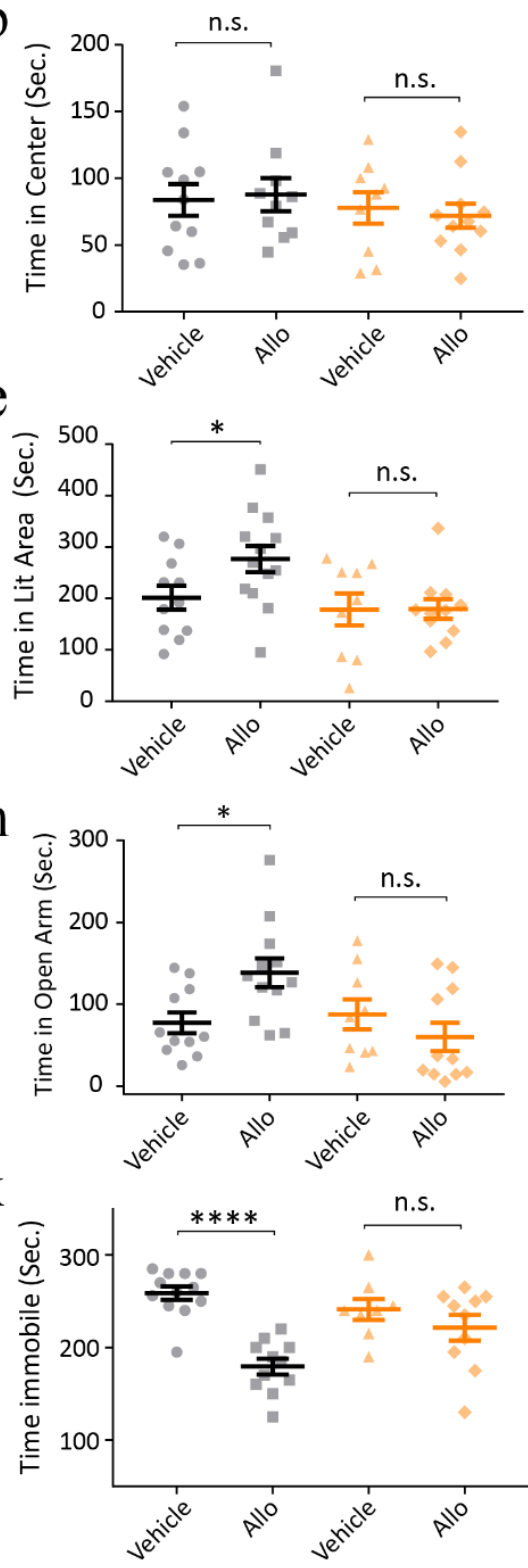

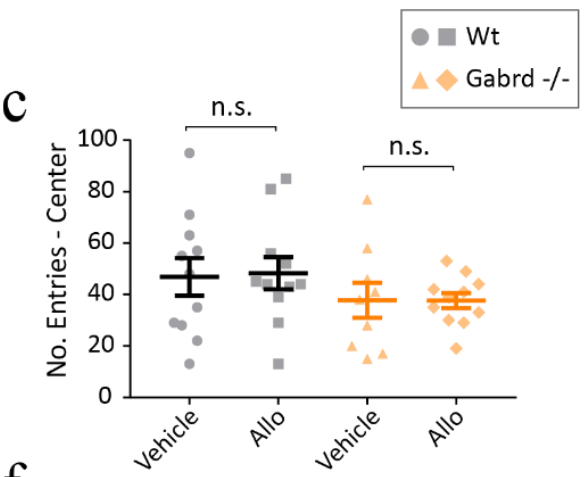

f

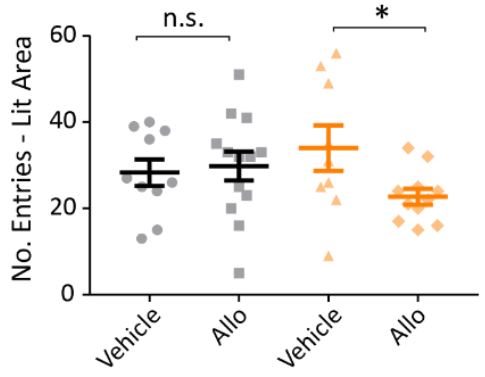

i
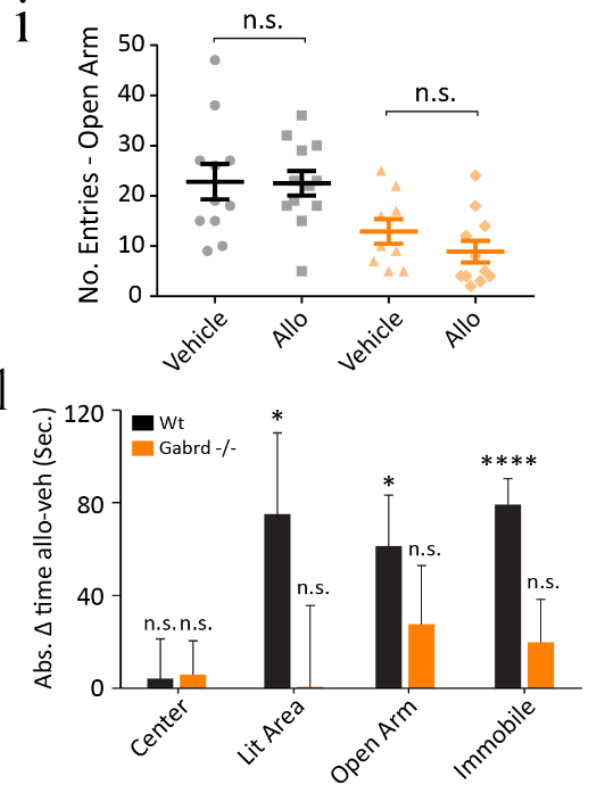

Figure 6. Allopregnanolone promoted anxiolysis in control but not in $\mathrm{Gabrd}^{-/}$mice. Schematics for behavioral tests and associated phenotypes in Wt mice infused with vehicle or $5 \mu \mathrm{g}(2.5 \mu \mathrm{g} / \mu \mathrm{l}$-inject) Allo: a, open field; $\mathbf{d}$, light dark box; $\mathbf{g}$, elevated plus maze, $\mathbf{j}$, Tail suspension test. Time that animals spend in b, center; e, lit area; $\mathbf{h}$, open arms, or $\mathbf{k}$, immobile. Number of Entries in c, center; $\mathbf{f}$, lit area; i, open arms. I, Summary of average absolute time difference in allo vs vehicle infused conditions across behavioral tests. Error bars represent SEM. Brackets and stars represent unpaired t-tests between vehicle vs allo treated groups. 
a

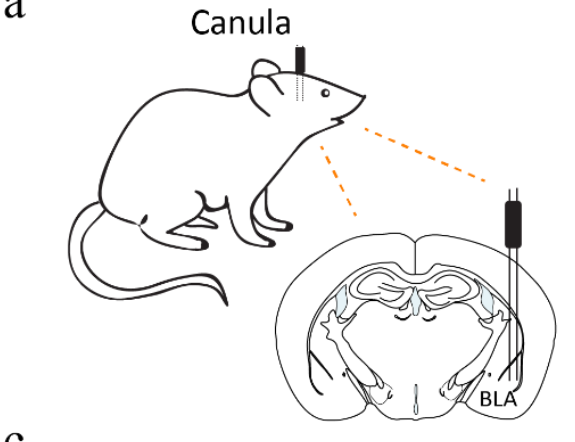

$\mathrm{c}$

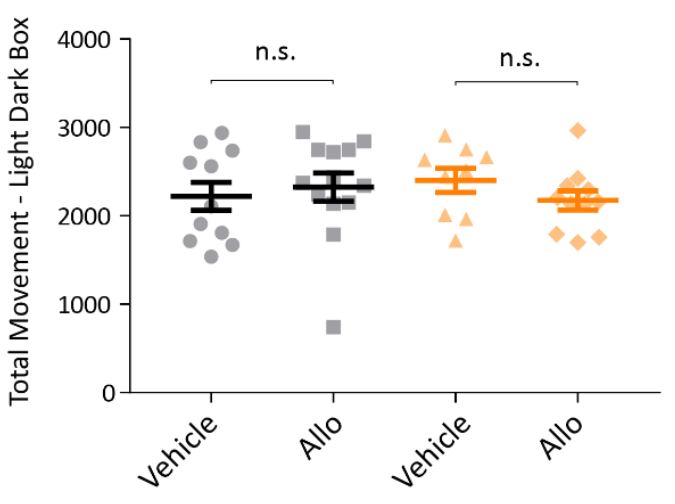

b

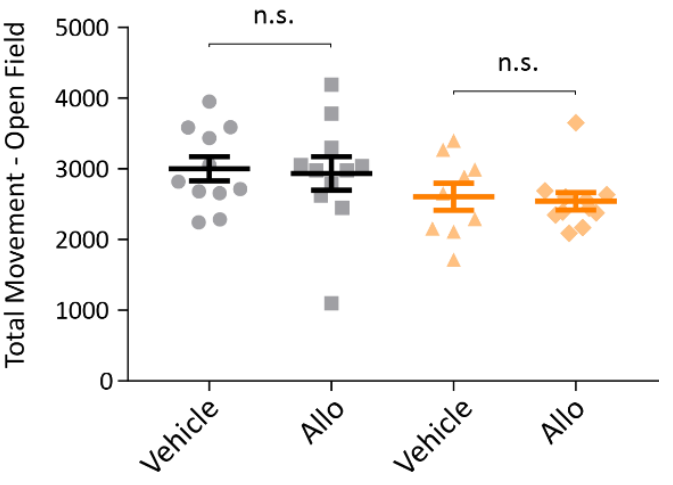

Supplementary Figure 5 (Supporting Figure 6). a, Schematic for canula implantation in basolateral amygdala (BLA). Total movement (\# total beam breaks) in b, Open Field c, Light Dark Box. Error bars represent SEM. Brackets represent unpaired t-tests between vehicle against allo treated groups. 
344 SGE-516 prevents depressive-like behavior induced by chronic unpredictable

\section{stress}

Our data show that neuroactive steroids alter behavioral states in WT mice. To assess the impact

347 of neuroactive neurosteroids on sustained threat, WT mice were exposed to chronic unpredictable stress (CUS). Animals were subjected to 3 consecutive weeks of alternating stressors (see methods). LFP and behavioral tests were performed prior to and 4 weeks post CUS induction (Fig. 7a). The 4 weeks post CUS timepoint was chosen due to the demonstrated sustainment of allo efficacy at least 30 days post-injection in phase 3 clinical trials of post-partum depression(Meltzer-Brody et al. 2018).

Mice that underwent CUS spend more time immobile in the tail suspension test (Fig. 7b; multiple comparison test) and had a lower preference for sucrose than control mice (Fig. 7b; Control: $85.3 \pm 2.72 \%, n=15$ animals; CUS: $70.0 \pm 4.41 \%, n=10$ animals; $p=0.0053$, Tukey's multiple comparison test). SAGE-516 application during CUS had the opposite effects as it decreased time immobile in the tail suspension test (Fig. 7b; CUS + SAGE-516: $146.3 \pm 7.49 \mathrm{~s}, \mathrm{n}=12$ animals, p<0.0001, Tukey's multiple comparison test) and elevated sucrose preference (Fig. 7b; CUS +

361 in controls (Fig. 7b). These results suggest that sustained threat through CUS induces behavioral 362 deficits in WT mice which are prevented by SGE-516 treatment. To investigate how BLA network activity is associated with behavioral changes, we compared BLA high theta $(6-12 \mathrm{~Hz})$ power change prior to and post CUS induction with time spend immobile in the tail suspension test. We

365 found that mice that underwent CUS had a positive non-significant correlation (Fig. 7c; $r=0.53$, 
$\mathrm{n}=10$ animals, $\mathrm{p}=0.11$, Pearson correlation) between time immobile and high theta change.

However, there was a significant negative correlation between (Fig. 7c; $r=-0.62, \mathrm{n}=11$ animals, $p=0.041$, Pearson correlation) time immobile and high theta change in CUS mice that were treated with SGE-516, indicating that larger increases in high theta power were associated with less time spend immobile and depressive-like behavior in mice $(z=2.54, p=0.01$, Fisher transformation). Furthermore, when including animals with the most extreme changes in behavioral outcomes from both CUS and CUS+SAGE-516 groups, there was a strong negative correlation (Fig. $7 \mathrm{~d} ; r=-0.84, \mathrm{n}=12$ animals, $\mathrm{p}=0.00057$, Pearson correlation) between time spent

374 immobile and change of high/low $(6-12 / 2-5 \mathrm{~Hz})$ theta power. These data suggest that larger high/low theta power increases in BLA are associated with improved behavioral outcomes.

379 following CUS exposure robustly reduced the total time spent immobile during a tail suspension

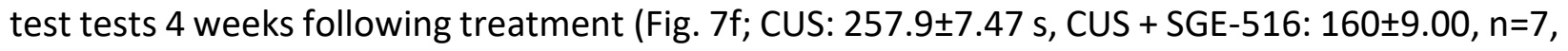
$p=0.0003$, paired t-test). Therefore, our data suggest that treatment with allo analog SGE-516 reduces behavioral deficits induced by sustained threat. Moreover, in CUS mice treated with SGE516 increases in high/low theta power from prior to post-CUS were associated with reduced time spent immobile in the tail suspension test (Fig. $7 g ; r=-0.74, n=8$ animals, $p=0.035$, Pearson 385 correlation). In addition, increased high/low theta ratio change in BLA (post-CUS/pre-CUS) was associated with the behavioral response elicited from SGE-516 treatment (Fig. 7h; $r=-0.86, \mathrm{n}=8$ 
388 theta ratio are associated with a reduction in behavioral deficits resulting from sustained threat

389 in mice.

390 To more broadly and unbiasedly examine the effects of CUS on global brain connectivity

391 we acquired the $\mathrm{fMRI}$ blood-oxygen-level-dependent (BOLD) signal. Mice that underwent the

392 CUS protocol exhibited altered functional connectivity across multiple brain areas when

393 compared to controls (Fig. 8i-j; CUS altered 84.95\% of control connections: 254/299 and 325 new

394 connections). Interestingly, SGE-516 treatment during CUS reversed $47.32 \%(274 / 579)$ of the CUS

395 altered connections restoring control connectivity (Fig. 8j). Additionally, 89.78\% (246/274) of the

396 reversed network connections were baseline control connections, indicating most of the new

397 connections made by CUS were not affected by SGE-516 treatment. These data show that

398 sustained threat produces brain-wide changes in functional connectivity of WT mice and suggest

399 that treatment with a synthetic neurosteroid analog of allo could act to partially restore those

400 changes. 
bioRxiv preprint doi: https://doi.org/10.1101/2021.03.08.434156; this version posted March 9, 2021. The copyright holder for this preprint (which was not certified by peer review) is the author/funder, who has granted bioRxiv a license to display the preprint in perpetuity. It is made available under aCC-BY-NC-ND 4.0 International license.

a<smiles>C1CCCCC1</smiles>

+ SGE-516

LFP session Behavioral testing

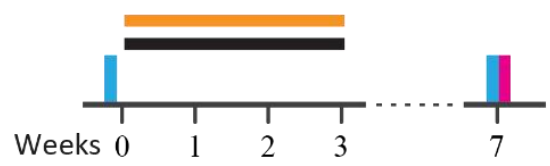

C

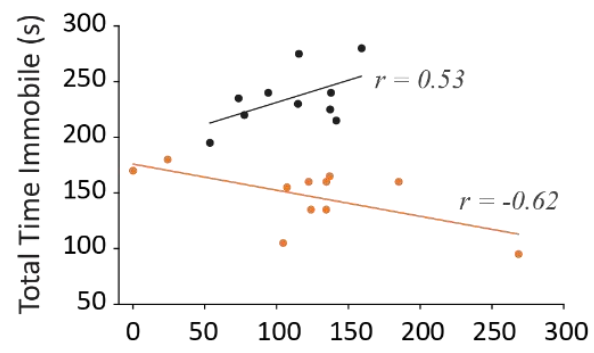

e

$\%$ Change Power Area $(6-12 \mathrm{~Hz})$

CUS + SGE-516

LFP session Behavioral testing

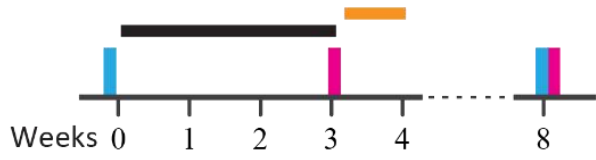

g

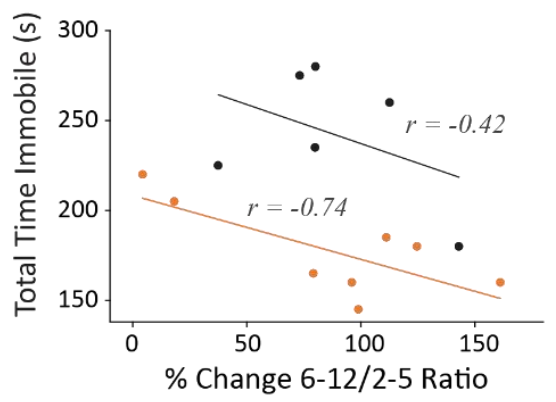

i

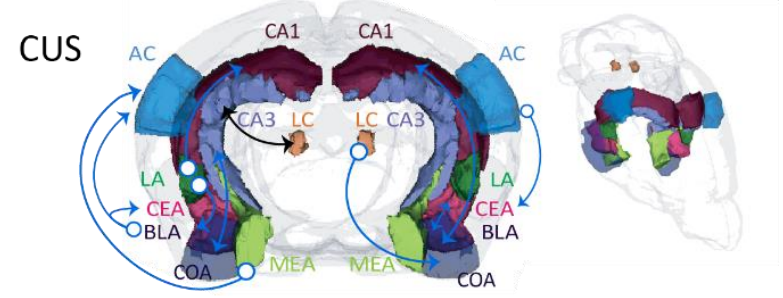

+ SGE-516

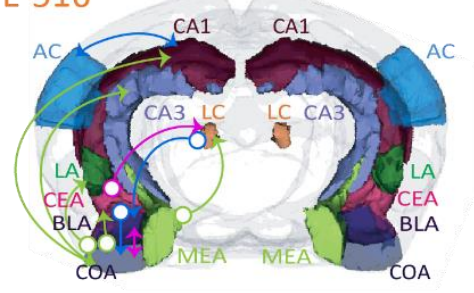

401
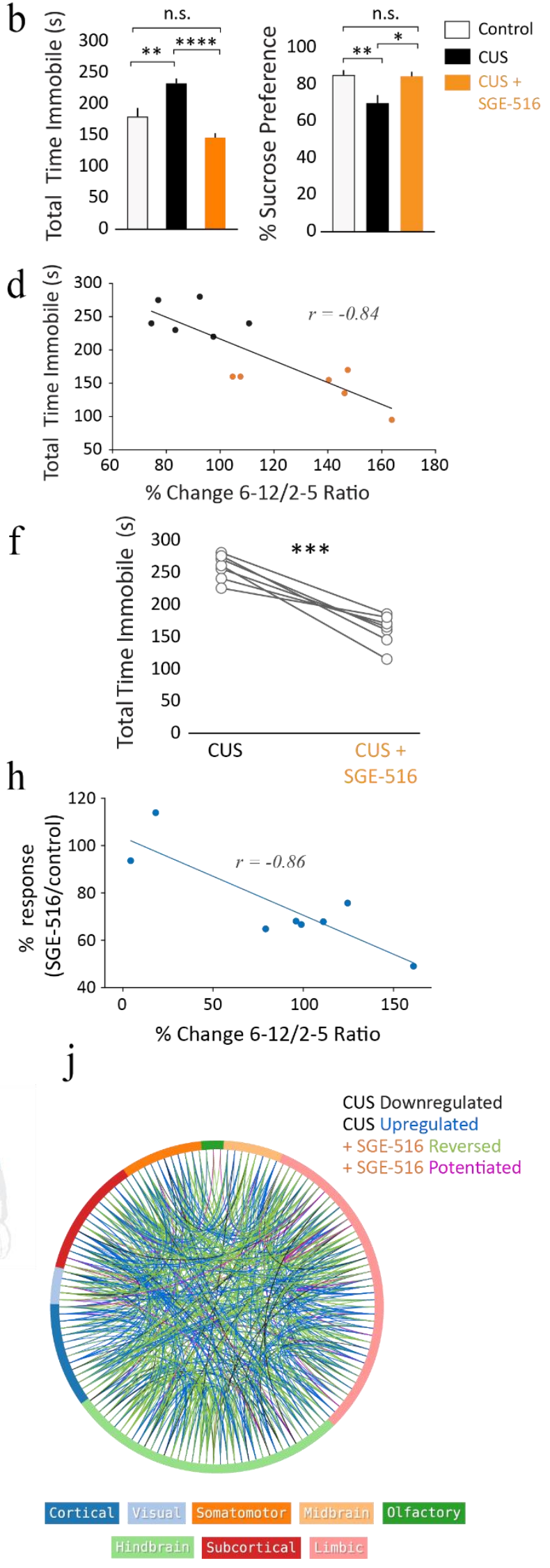
Figure 7. Synthetic Allopregnanolone analog SGE-516 prevents depressive-like behavior induced by chronic unpredictable stress (CUS) in C57bl/6 mice. a-d, SGE-516 application during CUS. a, Timeline of CUS paradigm with LFP and behavioral sessions. $\mathbf{b}$, Left - Time spent immobile in tail suspension test (TST) and Right- \% sucrose preference in control/no CUS (white), CUS (black), and CUS +SGE-516 (orange) animals. c, Time spent immobile in TST plotted against high theta $(6-12 \mathrm{~Hz})$ power change prior to and post CUS induction in CUS (black) and CUS + SGE-516 (orange) mice. $\mathbf{d}$, Time spent immobile in TST plotted against theta ratio change $(6-12 / 2-5 \mathrm{HzHz})$ in both CUS (black) and CUS +SGE-516 (orange) using outlier selection. e-h, SGE-516 application after CUS. e, Timeline of CUS paradigm with LFP and behavioral sessions. f, Time spent immobile in TST before and after SGE-516 application. $\mathbf{g}$, Time spent immobile in TST plotted against theta ratio $(6-12 / 2-5 \mathrm{HzHz})$ change prior to and post CUS induction in CUS (black) and CUS + SGE-516 (orange) mice. $h$, TST response difference prior to and post SGE-516 application plotted against theta ratio $(6-12 / 2-5 \mathrm{HzHz})$ change prior to and post CUS. Power changes in correlation plots were calculated as the percent difference between 4 weeks post-CUS and 24 hours prior to CUS; $r$-values represent the Pearson correlation coefficient. $\mathbf{i}-\mathbf{j}$, Resting state functional connectivity; type-of-connection legend: black: downregulated, blue: upregulated, green: reversed by SGE-516, pink: potentiated by SGE-516. i, Representative brain volumes of resting state functional connectivity in CUS and CUS+SGE-516 mice. Arrows indicate directionality of connections from the indicated seed regions; arrow color reflects type of connection change based on type-of-connection legend; AC: Auditory Cortex, BLA: Basal Lateral Amygdaloid Area, CA1: Cornu Ammonis 1, CA3: Cornu Ammonis 3, CEA: Central Nucleus of the Amygdala, COA: Cortical Amygdaloid Area, LA: Lateral Amygdaloid Area, LC: Locus Coeruleus, MEA: Medial Amygdaloid Area. j, Connectome map indicating significant changes in BOLD signal connectivity, Outer circle lines represent brain areas of the respective color as indicated in the bottom legend. 


\section{Discussion}

411 Here we demonstrate that neurosteroids alter network oscillations across multiple species (Fig.1)

412 and in rodents they potentiate high-theta ${ }^{6-12 \mathrm{~Hz}}$ power unlike other GABA agonists (Fig.1-2 \&

413 Supplementary Fig.1). This increase in BLA high-theta power was found to be largely mediated

414 through $\delta$ subunit-containing $\mathrm{GABA}_{\mathrm{A}} \mathrm{Rs}$ which are uniquely expressed in PV+ interneurons in the

415 BLA (Fig.2-3). Furthermore, the BLA network ex-vivo can self-sustain oscillations that are under

$416 \mathrm{PV}^{+}$interneuron control (Fig.4-5). Importantly, we show that acute allo infusion into the BLA is

417 sufficient to alter behavioral states (Fig. 6) and systemic neurosteroid (SGE-516) treatment

418 protects mice from behavioral deficits induced from sustained threat (Fig. 7).

It has been shown that distinct oscillatory states in BLA-PFC areas seem to be associated

with aversion and safety(Davis et al. 2017; Karalis et al. 2016; Likhtik et al. 2014). Indeed, the

421 behavioral expression of fear and safety associated with shifts in the low- ${ }^{\sim 4 \mathrm{~Hz}}$ and high-theta ${ }^{\sim \mathrm{Hz}}$

422 bands, respectively(Davis et al. 2017; Karalis et al. 2016; Ozawa et al. 2020). Here, we observed

that acute allo increased the high/low theta ${ }^{6-12 / 2-5 \mathrm{~Hz}}$ power in the BLA (Fig. 2f) and decreased

424 behaviors relevant to anxiety- and depressive-like states (Fig. 6). Furthermore, allo-analog (SGE-

425 516) treatment restored healthy behavioral states in mice that underwent sustained threat (Fig.

426 7e-f), where larger restoration was associated with greater increases in BLA high/low theta ${ }^{6-12 / 2-}$

$427{ }^{5 H z}$ power (Fig. 7g-h). Therefore, our findings reinforce the evidence that increases in high/low

428 theta band are associated with safety(Davis et al. 2017; Ozawa et al. 2020) and further suggest

429 that they could generalize them to healthy brain states beyond the realm of fear. We also

430 observed that high-theta ${ }^{6-12 \mathrm{~Hz}}$ and beta ${ }^{15-30 \mathrm{~Hz}}$ oscillations in BLA were significantly higher during

431 allo application in WT compared to $\mathrm{Gabrd}^{-1-}$ mice (Fig. 2e) indicating that they could also be an 
432 important component of neurosteroid treatment. Beta ${ }^{15-30 \mathrm{~Hz}}$ power was also strongly elevated

433 by diazepam in both mice and rats (Supplementary Fig. 1), in agreement to previous findings(Van

434 Lier et al. 2004). These results could suggest that high-theta ${ }^{6-12 \mathrm{~Hz}}$ and not beta ${ }^{15-30 \mathrm{~Hz}}$-oscillation

435 elevation is unique to the proposed mechanisms of action mediated by neuroactive steroids and

436 potentially contribute to their superior antidepressant effects.

We also examined whether the BLA network can support rhythmic oscillations. To the

best of our knowledge, we are the first to show that the mouse BLA can generate ex-vivo oscillations at gamma-band range ${ }^{\sim 40 \mathrm{~Hz}}$ during high $\mathrm{K}^{+} /$Kainate $\left(\mathrm{K}^{+} / \mathrm{KA}\right)$ tone (Fig. 4, Supplementary

Fig. 3). Gamma power is stronger in the BLA than nearby regions in awake mice, and BLA multiunits are phase-locked to BLA gamma oscillations(Kanta, Pare, and Headley 2019). Together,

442 these findings indicate that the BLA can generate and self-sustain network oscillations in the

443 gamma-band range despite the lack of an apparent laminar organization with parallel pyramidal

444 cell organization. The generation of gamma rhythmogenesis in the ex-vivo BLA seems to rely

445 exclusively on GABAergic interneuron signaling as these gamma-oscillations were only blocked

446 by a $\mathrm{GABA}_{\mathrm{A}} \mathrm{R}$ blocker and not by an AMPAR blocker (Fig. $4 \mathrm{~d}-\mathrm{g}$ ), in agreement to gamma

447 oscillations in the rat BLA(Randall, Whittington, and Cunningham 2011). Furthermore, prolonged

448 exposure of a $\mathrm{GABA}_{\mathrm{A}}$ receptor antagonist induced oscillations in the theta band-range ${ }^{3-12 \mathrm{~Hz}}$ (Fig.

$4494 \mathrm{f}-\mathrm{g})$. Therefore, it is possible that under certain conditions, such as reduced inhibitory tone, the

450 BLA can generate theta-oscillations intrinsically that can be detected by LFP recordings.

452 network activity in BLA slices (Fig. 5e-f, Supplementary Fig. 4b,c). PV interneurons form a large 
454 onto the perisomatic regions of their post-synaptic neurons, allowing them to effectively control

455 their output(Bartos, Vida, and Jonas 2007; Veres, Nagy, and Hájos 2017). Indeed, it has been

456 shown that rhythmic optogenetic BLA PV+ activation in awake mice entrains BLA multi-unit

457 activity(Ozawa et al. 2020). Thus, our findings together with existing literature suggest that $\mathrm{PV}^{+}$

458 interneurons are ideally poised to synchronize and control the oscillatory state of the BLA

459 network and pharmacological agents targeting this cellular mechanism are capable of altering

460 network and behavioral states.

We further observed that allo application enhances tonic inhibition on BLA $\mathrm{PV}^{+}$

462 interneurons but not on principal cells (Fig. 3), similar to previous reports(Liu et al. 2014). Tonic

463 inhibition on interneurons has been previously proposed to promote network

464 synchronization(Pavlov et al. 2014). It is possible that the ability of allo to modulate BLA network

465 synchronization involves the potentiation of tonic inhibition on PV+ interneurons.

The current study focused on the impact of allopregnanolone on oscillations within the

467 BLA. Future studies are required to resolve the impact of allopregnanolone on coupling between

468 anxiety-related networks, such as communication between the BLA and medial prefrontal cortex

469 or the ventral hippocampus(Calhoon and Tye 2015; Likhtik et al. 2014; Tovote, Fadok, and Lüthi

470 2015). Further, the impact of the saline injections on oscillations in the BLA, which interestingly

471 oppose the effects of allopregnanolone (Fig. 2c \& Supplementary Fig. 2a-b), are likely the result

472 of the stress of the injection and suggest that acute stress may also impact network and

473 behavioral states.

It was observed that the long-lasting antidepressant effect in clinical trials which outlasts

475 the pharmacokinetics of the drug exposure and is not easily explained by the known mechanism 
476 of action (Daly et al. 2018; Meltzer-Brody et al. 2018). Our data demonstrate that

477 allopregnanolone is able to shift the network oscillation state in the BLA through $\delta$ subunit-

478 containing $\mathrm{GABA}_{A} \mathrm{Rs}$, though not exclusively through this mechanism (Fig. 2, Fig. 7j). One could

479 envision that allopregnanolone acts on $\delta$ subunit-containing $\mathrm{GABA}_{\mathrm{A}} \mathrm{Rs}$ to shift the network to a

480 healthy network state that is more stable and can persist in the absence of the compound. Future

481 studies are required to fully understand the long-term effects of allopregnanolone on the

482 network, which will be informative for understanding the mechanisms mediating the clinical

483 effectiveness of allopregnanolone as an antidepressant treatment. These data demonstrate a

484 novel molecular and cellular mechanism orchestrating network and behavioral states, although,

485 we do not presume that this is the only mechanism involved in switching between network

486 states. In fact, there are likely numerous mechanisms which can alter the network in a similar

487 manner(Swensen and Marder 2000).

488

489

490

491

492

493

494

495

496

497 


\section{References}

Antonoudiou, Pantelis et al. 2020. "Parvalbumin and Somatostatin Interneurons Contribute to the Generation of Hippocampal Gamma Oscillations." Journal of Neuroscience 40(40): 7668-87. https://doi.org/10.1523/JNEUROSCI.0261-20.2020 (February 1, 2021).

Babaev, Olga, Carolina Piletti Chatain, and Dilja Krueger-Burg. 2018. "Inhibition in the Amygdala Anxiety Circuitry." Experimental and Molecular Medicine 50(4).

Bartos, Marlene, Imre Vida, and Peter Jonas. 2007. "Synaptic Mechanisms of Synchronized Gamma

Calhoon, Gwendolyn G., and Kay M. Tye. 2015. "Resolving the Neural Circuits of Anxiety." Nature Neuroscience 18(10): 1394-1404.

Daly, Ella J. et al. 2018. “Efficacy and Safety of Intranasal Esketamine Adjunctive to Oral Antidepressant Therapy in Treatment-Resistant Depression: A Randomized Clinical Trial." JAMA Psychiatry 75(2):

Davis, Patrick, Yosif Zaki, Jamie Maguire, and Leon G Reijmers. 2017. “Cellular and Oscillatory Substrates of Fear Extinction Learning." Nature Neuroscience 20(11): 1624-33. http://www.nature.com/articles/nn.4651 (August 2, 2019).

Felix-Ortiz, A. C. et al. 2016. "Bidirectional Modulation of Anxiety-Related and Social Behaviors by Amygdala Projections to the Medial Prefrontal Cortex." Neuroscience 321: 197-209.

Fenster, Robert J., Lauren A.M. Lebois, Kerry J. Ressler, and Junghyup Suh. 2018. “Brain Circuit 19(9): 535-51.

Jobert, Marc et al. 2012. "Guidelines for the Recording and Evaluation of Pharmaco-EEG Data in Man:

521 https://www.karger.com/Article/FullText/343478 (March 9, 2020). 
522

523

524

525

526

527

528

529

530

531

532

533

534

535

536

537

538

Kanta, Vasiliki, Denis Pare, and Drew B. Headley. 2019. "Closed-Loop Control of Gamma Oscillations in the Amygdala Demonstrates Their Role in Spatial Memory Consolidation." Nature Communications 10(1): 1-14.

Karalis, Nikolaos et al. 2016. "4-Hz Oscillations Synchronize Prefrontal-Amygdala Circuits during Fear Behavior." Nature Neuroscience 19(4): 605-12.

Krabbe, Sabine, Jan Gründemann, and Andreas Lüthi. 2018. “Amygdala Inhibitory Circuits Regulate Associative Fear Conditioning." Biological psychiatry 83(10): 800-809. http://www.ncbi.nlm.nih.gov/pubmed/29174478 (August 2, 2019).

Lee, Vallent, and Jamie Maguire. 2014. "The Impact of Tonic GABAA Receptor-Mediated Inhibition on Neuronal Excitability Varies across Brain Region and Cell Type." Frontiers in Neural Circuits 8(FEB).

Van Lier, Hester, Wilhelmus H.I.M. Drinkenburg, Yvonne J.W. Van Eeten, and Anton M.L. Coenen. 2004. "Effects of Diazepam and Zolpidem on EEG Beta Frequencies Are Behavior-Specific in Rats." Neuropharmacology 47(2): 163-74.

Likhtik, Ekaterina et al. 2014. "Prefrontal Entrainment of Amygdala Activity Signals Safety in Learned Fear and Innate Anxiety." Nature Neuroscience 17(1): 106-13.

Liu, Zhi Peng et al. 2014. “Chronic Stress Impairs GABAergic Control of Amygdala through Suppressing the Tonic GABAA Receptor Currents." Molecular Brain 7(1): 32. http://molecularbrain.biomedcentral.com/articles/10.1186/1756-6606-7-32 (February 27, 2020).

Meltzer-Brody, Samantha et al. 2018. "Brexanolone Injection in Post-Partum Depression: Two Multicentre, Double-Blind, Randomised, Placebo-Controlled, Phase 3 Trials." The Lancet 392(10152): 1058-70.

Mihalek, Robert M. et al. 1999. "Attenuated Sensitivity to Neuroactive Steroids in Y-Aminobutyrate Type A Receptor Delta Subunit Knockout Mice." Proceedings of the National Academy of Sciences of the United States of America 96(22): 12905-10. 
Muller, Jay F., Franco Mascagni, and Alexander J. McDonald. 2005. “Coupled Networks of ParvalbuminImmunoreactive Interneurons in the Rat Basolateral Amygdala." Journal of Neuroscience 25(32): 7366-76.

Ozawa, Minagi et al. 2020. “Experience-Dependent Resonance in Amygdalo-Cortical Circuits Supports Fear Memory Retrieval Following Extinction." Nature Communications 11(1): 1-16. https://doi.org/10.1038/s41467-020-18199-w (February 2, 2021).

Pavlov, Ivan et al. 2014. "Tonic GABAA Conductance Bidirectionally Controls Interneuron Firing Pattern and Synchronization in the CA3 Hippocampal Network." Proceedings of the National Academy of Sciences of the United States of America 111(1): 504-9.

Randall, Fiona E., Miles A. Whittington, and Mark O. Cunningham. 2011. "Fast Oscillatory Activity Induced by Kainate Receptor Activation in the Rat Basolateral Amygdala in Vitro." European Journal of Neuroscience 33(5): 914-22. http://doi.wiley.com/10.1111/j.1460-9568.2010.07582.x (February $26,2020)$.

Schiller, Crystal Edler, Peter J. Schmidt, and David R. Rubinow. 2014. "Allopregnanolone as a Mediator of Affective Switching in Reproductive Mood Disorders." Psychopharmacology 231(17): 3557-67.

Stell, Brandon M. et al. 2003. "Neuroactive Steroids Reduce Neuronal Excitability by Selectively Enhancing Tonic Inhibition Mediated by $\delta$ Subunit-Containing GABAA Receptors." Proceedings of the National Academy of Sciences of the United States of America 100(SUPPL. 2): 14439-44.

Stujenske, Joseph M., Ekaterina Likhtik, Mihir A. Topiwala, and Joshua A. Gordon. 2014. "Fear and Safety Engage Competing Patterns of Theta-Gamma Coupling in the Basolateral Amygdala." Neuron 83(4): 919-33.

Swensen, Andrew M., and Eve Marder. 2000. "Multiple Peptides Converge to Activate the Same VoltageDependent Current in a Central Pattern-Generating Circuit." Journal of Neuroscience 20(18): 675259. 
bioRxiv preprint doi: https://doi org/10.1101/2021.03.08.434156; this version posted March 9, 2021. The copyright holder for this preprint (which was not certified by peer review) is the author/funder, who has granted bioRxiv a license to display the preprint in perpetuity. It is made available under aCC-BY-NC-ND 4.0 International license.

570 Tovote, Philip, Jonathan Paul Fadok, and Andreas Lüthi. 2015. “Neuronal Circuits for Fear and Anxiety."

571 Nature Reviews Neuroscience 16(6): 317-31.

572 Veres, Judit M, Gergő A Nagy, and Norbert Hájos. 2017. “Perisomatic GABAergic Synapses of Basket Cells

$573 \quad$ Effectively Control Principal Neuron Activity in Amygdala Networks." eLife 6.

574 https://elifesciences.org/articles/20721 (August 2, 2019).

575

576

577

578

579

580

581

582

583

584

585

586

587

588

589 


\section{Author Contributions}

591 Conceptualization \& Writing-Original: P.A and J.L.M., Project Administration, Supervision \&

592 Funding Acquisition: J.L.M., Visualization: P.A and P.L.W.C, Data curation, programming and

593 mouse LFP analysis: P.A., Writing-Review \& Editing: P.A, J.L.M, P.L.W.C, N.L.W, G.L.W, A.C.S, D.P.N, M.K. and M.C.Q.

J.L.M conducted and analyzed whole-cell, mouse awake LFP and behavior experiments, P.A and

P.L.W.C conducted ex-vivo LFP experiments, A.C.S, D.P.N, M.K. and M.C.Q conducted and analyzed the fMRI experiments.

\section{Acknowledgements}

602 The authors would like to thank Dr. Leon Reijmers for providing constructive comments on the 603 manuscript. The authors would also like to thank the Biostatistics, Epidemiology, and Research 604 Design (BERD) Center and specifically Dr. Jessica Paulus, ScD, for consultation and assistance 605 with the correlational analysis and statistical measures. Collectively we also thank the Center 606 for Translational Neuroimaging at Northeastern University for providing access to the Bruker 607 BioSpec 7.0T and for assisting in fMRI data collection and analysis. J.L.M., P.A., N.L.W, G.L.W, P.L.W.C., and L.C.M. were supported by R01AA026256, R01NS105628, R01NS102937, and a sponsored research agreement with SAGE Therapeutics. 
bioRxiv preprint doi: https://doi.org/10.1101/2021.03.08.434156; this version posted March 9, 2021. The copyright holder for this preprint (which was not certified by peer review) is the author/funder, who has granted bioRxiv a license to display the preprint in perpetuity. It is made available under aCC-BY-NC-ND 4.0 International license.

612 J.M. serves as a member of the Scientific Advisory Board for SAGE Therapeutics, Inc. A.S., D.N.,

613 M.L, and M.Q. are employees of SAGE Therapeutics, Inc., 